\title{
Temperaturas mínimas estivales en el sureste de la península ibérica a partir de termografías satelitales
}

\section{Minimum summer temperatures in the southeast of the iberian peninsula through satellite thermographies}

\author{
David Espín-Sánchez \\ david.espin1@um.es@0000-0003-4807-5450 \\ Departamento de Geografía, Facultad de Letras, Universidad de Murcia. \\ C/ Santo Cristo. 30001 Murcia, España.
}

\section{INFO ARTÍCULO}

Recibido: 11-12-2020

Revisado: 15-04-2021

Aceptado: 22-04-2021

\section{PALABRAS CLAVE}

Gradiente altitudinal Inversión térmica

Polo frío

Termografía

Canícula

Temperaturas mínimas

Sureste peninsular

Teledetección

\begin{abstract}
RESUMEN
La posición latitudinal del sureste de la península ibérica genera temperaturas medias elevadas (entre 25 y $30^{\circ} \mathrm{C}$ ) durante la época estival, si bien, su compleja orografía y el importante gradiente altitudinal, genera importantes diferencias en las temperaturas mínimas, influenciadas especialmente por intensos procesos de inversiones térmicas nocturnas (ITN) en valles de interior. El incremento de nuevas estaciones meteorológicas y el aumento de la densidad de las redes de observación, unida a la implantación de una red propia de termómetros registradores en polos fríos (CAP) de las provincias de Murcia, Albacete o Jaén, lleva a un mayor conocimiento de la distribución de temperaturas nocturnas superficiales. El principal objetivo de la investigación es la generación de cartografía (termografías) que refleje la distribución de las temperaturas nocturnas en el sureste de la Península Ibérica, realizada a través del tratamiento de productos satelitales (VIIRS LST Band I5 del satélite SUOMI-NPP. Unido al uso de las imágenes satelitales (termografías) es posible analizar detalladamente los contrastes térmicos existentes durante el periodo canicular a través de 147 estaciones meteorológicas y termómetros registradores ( 15 de julio al 15 de agosto) de los años 2017 a 2020. La verificación de las termografías utilizadas (producto Band I5) muestra un notable ajuste estadístico (R2 de 0,87), con un Bias y RMSE de $-0,2$ y $1,83^{\circ} \mathrm{C}$ respectivamente. La cartografía es realizada para las diferencias provincias que integran el sureste de la península ibérica (Alicante, Albacete, Murcia, Jaén, Granada y Almería). Las diferencias térmicas diarias aportadas por las estaciones meteorológicas superficiales arrojan contrastes de temperaturas mínimas de hasta $23,4^{\circ} \mathrm{C}$ entre valles elevados de interior y ámbitos litorales.
\end{abstract}

\section{KEYWORDS}

Lapse rate

Temperature inversion

Cold air pools

Thermography

Hottest days of summer

Sureste peninsular

Teledetection

\begin{abstract}
The latitudinal position of the southeast of the Iberian Peninsula generates high average temperatures (between 25 and $30^{\circ} \mathrm{C}$ ) during the summer season, although its complex orography and the important lapse rate (LR) give rise to important differences in minimum temperatures, influenced by intense processes of nocturnal temperature inversion (ITN) in inland valleys. The increase of new meteorological stations and the rise in the density of observation networks, together with the implementation of its own network in cold air pools (CAP) in the provinces of Murcia, Albacete or Jaén, leads to a greater understanding of the distribution of surface temperatures. The main objective of the research is the generation of cartography (thermographs) that reflects the distribution of night temperatures in the southeast of the Iberian Peninsula, carried out through the treatment of satellite products (VIIRS LST Band I5 of the SUOMI-NPP satellite). Together with the use of satellite images (thermography), it is possible to analyze in detail the thermal contrasts that exist during the hottest days of summer through 147 weather stations and recording thermometers
\end{abstract}




\begin{abstract}
(July 15 to August 15) of the year 2017 to 2020. Verification of the thermographs used (Band I5 product) shows a notable statistical adjustment (R2 of 0.87 ), with a Bias and RMSE of -0.2 and $1.83^{\circ} \mathrm{C}$ respectively. The cartography is carried out for the differences in the provinces that make up the SE of the Iberian Peninsula (Alicante, Albacete, Murcia, Jaén, Granada and Almería), with daily thermal differences in minimum temperatures of up to $23.4^{\circ} \mathrm{C}$ between inland valleys and coastal areas.
\end{abstract}

\title{
1. INTRODUCCIÓN
}

Una inversión térmica nocturna (ITN) supone un cambio térmico en el gradiente altitudinal, con temperaturas más bajas por debajo del estrato de inversión, y valores más elevados en un nivel superior. La deposición de aire más frío y denso en los fondos de valle y depresiones se lleva a cabo durante horas nocturnas de estabilidad atmosférica. El viento sinóptico débil y ausencia de nubosidad durante las madrugadas estables en zonas orográficamente deprimidas o de valle, provoca una pérdida de radiación y calor en la superficie del suelo (Whiteman et al., 2004), destruyéndose por convección después del amanecer (Arduini et al., 2020). Se generan las denominadas piscinas de aire frío (cold air pools) o CAP, una capa de aire estable y estratificada que se encuentra confinada en el fondo de un valle o cuenca. El proceso de enfriamiento local genera un estrato superficial de aire frío confinado y desacoplado del flujo sinóptico más cálido de niveles altitudinalmente superior (Sheridan, 2019).

Un gran número de investigaciones (Sauberer \& Dirmhirn, 1954, 1956; Litschauer, 1962, Whiteman et al., 2004, 2008; Eisenbach et al., 2003; Pospichal et al., 2003) han puesto de manifiesto la importante variabilidad espacial en las temperaturas mínimas sobre orografía irregular y compleja, y el acopio máximo de frío sobre plataformas kársticas y campos de dolinas, determinando, que es ahí, donde se registran las temperaturas más bajas del planeta, como los $-56^{\circ} \mathrm{C}$ en Peter Sink (Utah, EE.UU) o $-50^{\circ} \mathrm{C}$ en Gstettneralm (Austria).

La acumulación de aire frío en los fondos de la depresión se ve reforzada cuando actúan flujos catabáticos, potenciándose el proceso a razón del incremento del confinamiento de las tierras bajas (Gustavsson et al., 1998; Lindkvist et al., 2000; Lundquist et al., 2008). Así, como resultado de la influencia de estas variables climáticas y topográficas, la intensidad y el espesor de los fenómenos de inversión térmica se alteran de acuerdo con la situación sinóptica, la persistencia del balance energético negativo y la configuración topográfica del valle (McKee \& O'Neal, 1989; Whiteman, 2000; Clements et al., 2003; Daly et al., 2009).

Los extraordinarios contrastes térmicos entre los fondos de valle y ámbitos geográficamente accidentados lleva a la necesidad de poseer información espacial con un creciente interés científico en busca de nuevas metodologías satelitales para rellenar los vacíos climáticos existentes. Los recientes avances en los instrumentos de teledetección y potencia de cálculo, junto con mejoras en parametrizaciones y algoritmos numéricos, dan lugar a análisis detallados de posibles acumulaciones de aire frío en fondos de valle. Se demuestra, por tanto, que las ITN tienen un efecto muy notable sobre el clima, con formación persistente de estratos fríos superficiales (Zhang et al., 2011). La previsión en invierno de la acumulación y de aire frío persistentes se ve obstaculizada por la falta actual de comprensión en los mecanismos involucrados, y es considerado uno de los problemas más difíciles de pronosticar en cuencas y valles

En años recientes, los importantes avances en teledetección, han conseguido el desarrollo de nuevos algoritmos y ecuaciones para la obtención de temperatura del aire a 1,5 $\mathrm{m}$ de altura (Ta) a través de la temperatura de la superficie a $2 \mathrm{~m}$ (Ts), obtenida a partir de imágenes de sensores remotos satelitales (Vancutsem et al., 2010; Zhu et al., 2013). La Ta es un parámetro de gran importancia para una amplia gama de campos, como la agricultura (evapotranspiración de cultivos, predicción del rendimiento de cultivos, hidrología, ecología, medio ambiente y cambio climático).

Los productos LST de diferentes sensores (MODIS, SUOMI NPP, NOAA20, AVHRR...) se han utilizado con éxito en diversos ámbitos científicos, por ejemplo, para la estimación de la evapotranspiración (Anderson et al., 2011), estimación de la temperatura del aire (Vancutsem et al., 2010) y el monitoreo de islas de calor urbano (Rajasekar \& Weng, 2009). El uso de productos LST puede ayudar al desarrollo de registros de datos climáticos LST de resolución moderada a largo plazo (Yu et al., 2008). 
La gran resolución espacial y temporal (diaria), datos en abierto, y el fácil acceso a las imágenes satelitales, ha generado un importante incremento de los estudios que emplean la Ta (Noi et al., 2016; Sismanidis et al., 2016; Zhou et al., 2017), especialmente utilizando información del instrumento Visible Infrared Imaging Radiometer Suite (VIIRS) a bordo del satélite Suomi National Polar-Orbiting Partnership (Suomi NPP) (Hillger et al., 2013). También son muy utilizados los productos generados por el sensor Moderate Resolutions Imaging Spectroradiometer (MODIS6), que viaja a bordo de las plataformas satelitales Terra (1999) y Aqua (2002) (Wan et al., 2015).

Se han determinado correlaciones estadísticas muy notables $\left(R^{2}>0,90\right)$ y un sesgo mínimo (RMSE y MD $<7^{\circ} \mathrm{C}$ ) entre los productos que estiman Ta y la temperatura del aire a $2 \mathrm{~m}$ registradas por estaciones meteorológicas superficiales $(T)$, demostrando ser lo suficientemente precisos para la estimación de temperaturas mínimas superficiales multitud de ámbitos planetarios (Liu et al., 2015; Hulley et al., 2017; Niclòs et al., 2018).

La interpolación espacial ha sido ampliamente aplicada para la cartografía de temperaturas mínimas (Thornton et al., 1997; Jarvis \& Stuart, 2001), donde la mayoría de los modelos de interpolación espacial incorpora una corrección de gradiente vertical con el fin de adaptar la diferencia de elevación y evitar los importantes sesgos generados (Dodson \& Marks, 1997; Nalder \& Wein, 1998). El gradiente varía temporalmente a escalas estacionales, diarias e incluso horarias. Además, la ubicación de la estación de referencia puede afectar al gradiente vertical (Bolstad et al., 1998) causando un sesgo positivo para elevaciones más bajas. Por tanto, aunque el método de interpolación ha sido ampliamente aplicado para la estimación de temperaturas mínimas durante fenómenos de ITN, su limitación espacial y vertical limita su uso.

Para paliar las limitaciones de las técnicas de interpolación espacial de las últimas décadas (Dodson \& Marks, 1997), debido a la distribución desigual y escasa de los puntos de observación (Bolstad et al., 1998), se utilizan productos satelitales donde se recupera la temperatura superficial (LST). Para reflejar la distribución de temperaturas nocturnas en el sureste de la península ibérica, se genera cartografía (termografías) a través de productos satelitales, siendo uno de los principales objetivos de la investigación. Se analizan, también los contrastes térmicos en la época estadísticamente más cálida del año (periodo canicular de 2017 a 2020), con el apoyo de datos observacionales en estaciones meteorológicas y termómetros registradores, que son utilizados también para el segundo gran objetivo de la investigación, el de la validación del producto satelital en el área de estudio

\section{DATOS Y MÉTODOS}

\subsection{Datos}

El análisis de temperaturas mínimas superficiales se sustenta a través de la utilización de temperaturas mínimas diarias y horarias pertenecientes a 147 estaciones meteorológicas automáticas (EMA) (mapa 1) distribuidas espacialmente en el sureste de la península ibérica (provincias de Alicante, Albacete, Murcia, Almería, Granada y Jaén). Un total de 92 estaciones pertenecen al Sistema de Información Agroclimática para el Regadío (SIAR) del Ministerio de Agricultura, Pesca y Alimentación; 32 a la Red de estaciones agroclimáticas de la Consejería de Agricultura, Ganadería, Pesca y Desarrollo Sostenible de la Junta de Andalucía; 8 a la Agencia Estatal de Meteorología (AEMET), y finalmente 15 termómetros registradores pertenecientes a la red propia del autor (Espín-Sánchez et al., 2018b).

La utilización de la red propia de datalogger (cuadro 1) se justifica por la utilización de enclaves con temperaturas medias de las mínimas más bajas que las aportadas por las redes oficiales, en un esfuerzo de llegar a áreas pobladas de alta montaña y zonas remotas. Los termómetros registradores (dataloggers) utilizados son en su mayoría de marca Elitech RC-5 de 25.000 registros (con certificación de calibrado del CNAS, China National Accreditation Service), dos de ellos son Microlite 32K (32.000 registros), y finalmente, ubicados en los dos emplazamientos con las temperaturas medias más frías, y bajo condiciones invernales más severas, se utilizan dos registradores HOBO MX25 de 50.000 registros. En la mayoría de observatorios se utiliza una garita meteorológica artesanal compuesta por 11 platos blancos, en 3 de ellas la garita meteorológica homologada es tipo Davis (7714), y finalmente, para los termómetros ubicados en los parajes de Navalasno y Nava del Pol- 
vo se ubican en el interior de dos garitas meteorológicas tipo pagoda, cedidas por AEMET Murcia (imagen 1). Los registradores utilizados poseen un rango temporal diezminutal, con una precisión de $\pm 0,3^{\circ} \mathrm{C}$ y una resolución térmica de $0,1^{\circ} \mathrm{C}$. Los ubicados en Monterilla y Navalasno son calibrados por la Delegación Territorial de AEMET de Murcia, en el Centro Meteorológico Territorial de Guadalupe. Los responsables del mantenimiento del instrumental determinan una desviación de $+0,2^{\circ}$ en las temperaturas mínimas.

Las temperaturas mínimas diarias y de rango temporal diezminutal han sido analizadas exclusivamente para cuatro periodos caniculares (del 15 de julio al 15 de agosto) para los años 2017, 2018, 2019 y 2020. Desde el punto de vista estadístico, el periodo canicular posee la temperatura media más elevada del año (Mosiño \& García, 1966).

La realización de la cartografía de temperaturas nocturnas superficiales (termografías) ha sido elaborada a través del producto satelital VIIRS LST, obtenido del instrumento VIIRS, perteneciente al satélite SUOMI-NPP (https://worldview.earthdata.nasa.gov/).

Los productos satelitales del instrumento VIIRS se dividen en 22 bandas con una cobertura espectral de 0,4 a $12 \mu \mathrm{m}$. Entre las 22 bandas hay 5 canales de imágenes de alta resolución (bandas I), 16 canales de resolución moderada (bandas M) y banda de 1 día / noche (DNB). (Cao et al., 2013). Se utiliza específicamente el subproducto Band I5 (una capa de temperatura de brillo calcula a partir de radiancias calibradas de VIIRS VNP02), con una resolución espacial de 375 m, y una resolución temporal diaria (Cao et al., 2013). El sesgo de medición y la precisión especificada para el producto es de 1,4 y 0,5 K respectivamente, que deben cumplirse cuando la máscara de nube indica una alta confianza en condiciones claras (Minnett et al., 2014). Posee una órbita sincrónica solar a una altitud de $829 \mathrm{~km}$ y con unos tiempos de paso sobre la vertical del área de estudio entre las 1.40 y $2.40 \mathrm{~h}$ de la madrugada (Niclòs et al., 2018).

Para la validación estadística del producto VIIRS LST (Band I5) se utilizan las temperaturas superficiales de los observatorios meteorológicos anteriormente descritos, tomadas a las $2.00 \mathrm{~h}$ AM debido a su mayor proximidad con el paso del satélite SUOMI NPP sobre la vertical del área de estudio.

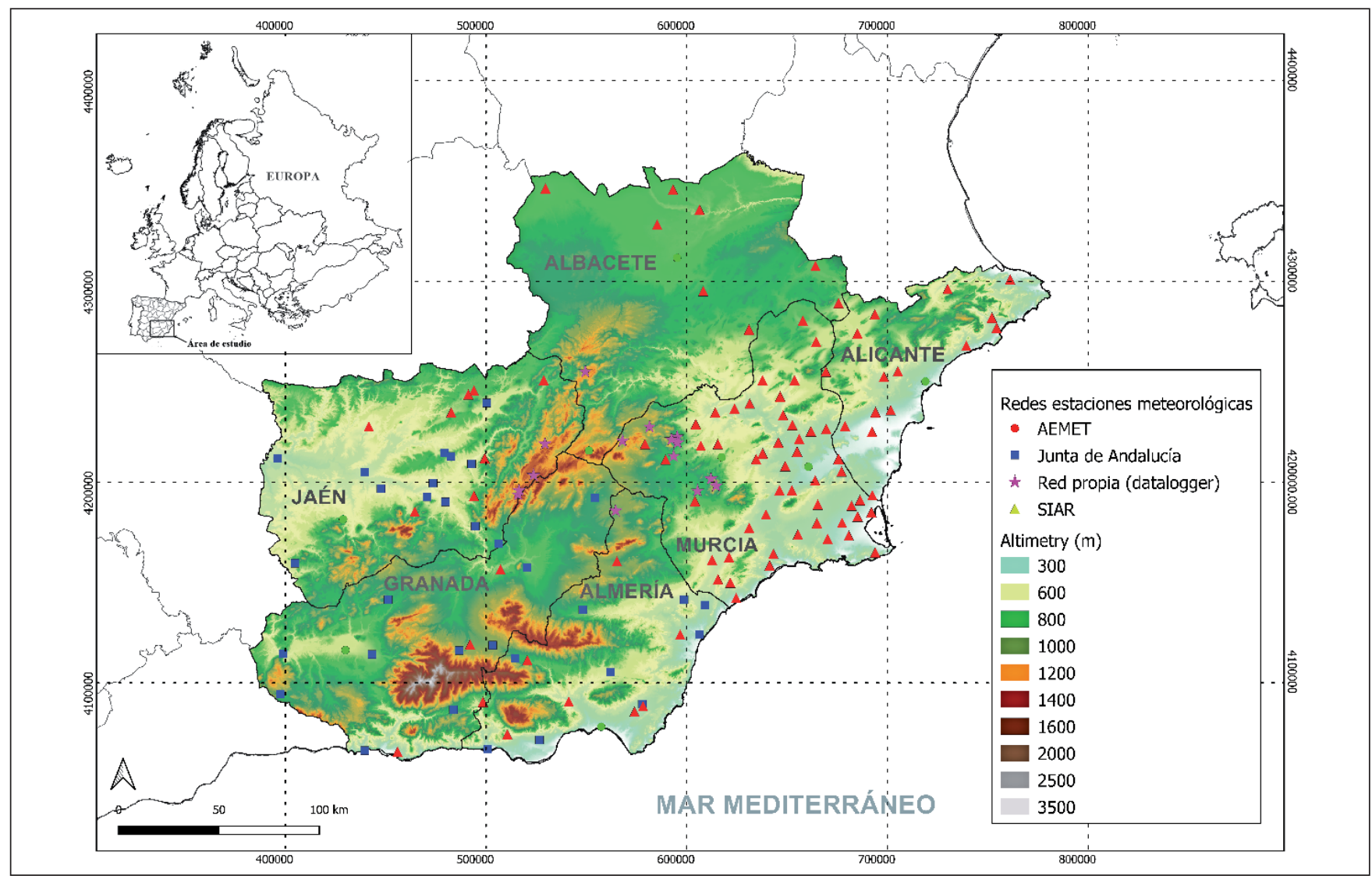

Mapa 1. Estaciones meteorológicas utilizadas en el área de estudio. Fuente: Elaboración propia. 
Cuadro 1. Características de los registradores de temperatura (dataloggers) de la red propia.

\begin{tabular}{|c|c|c|c|c|}
\hline Paraje & Provincia & Altitud & Protección & Sensor \\
\hline Nava del Polvo & Jaén & 1836 & Pagoda AEMET & HOBO MX25 \\
\hline Navalasno & Jaén & 1770 & Pagoda AEMET & Microlite 32K \\
\hline Monterilla & Jaén & 1660 & David 7714 & HOBO MX25 \\
\hline Viboreros & Albacete & 1555 & Garita casera & Elitech RC-5 \\
\hline Pico de la Selva & Murcia & 1521 & Garita Davis & Elitech RC-5 \\
\hline Vega de Pontones & Jaén & 1338 & Garita casera & Elitech RC-5 \\
\hline Nevazo de Arriba & Murcia & 1181 & Garita casera & Microlite 32K \\
\hline La Rogativa & Murcia & 1152 & Garita casera & Elitech RC-5 \\
\hline Cañada Grande & Murcia & 1087 & Garita casera & Elitech RC-5 \\
\hline La Risca & Murcia & 1047 & David 7714 & Elitech RC-5 \\
\hline Gollaín & Murcia & 1021 & Garita casera & Elitech RC-5 \\
\hline Bco. del Agua & Murcia & 906 & Garita casera & Elitech RC-5 \\
\hline Bco. del Cenajo & Murcia & 865 & Garita casera & Elitech RC-5 \\
\hline Benablón & Murcia & 777 & Garita casera & Elitech RC-5 \\
\hline Avilés & Murcia & 762 & Garita casera & Elitech RC-5 \\
\hline
\end{tabular}

Fuente: Elaboración propia.

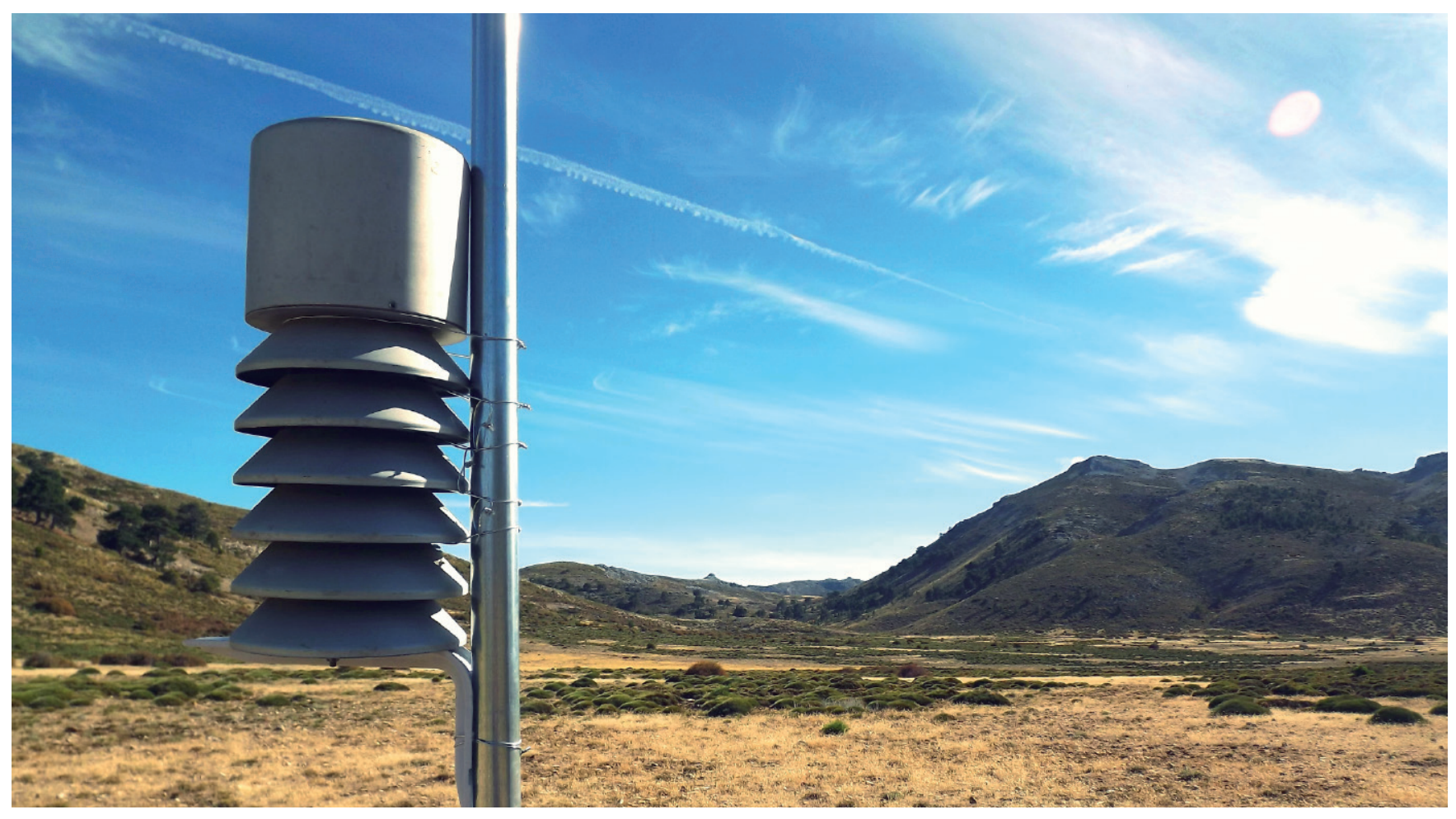

Imagen 1. Sensor térmico (datalogger) localizado en el poljé colgado de Navalasno (Jaén), a una altitud de 1770 m. Garita meteorológica de tipo Pagoda, cedida por AEMET Murcia, con sensor microlite 32K en su interior. Fuente: Autor. 


\subsection{Métodos}

El producto VIIRS LST (Band I5) utiliza el algoritmo de ventana dividida (Yu et al., 2005) recurriendo a la temperatura de brillo (Ti) medidas en dos bandas espectrales VIIRS: M15 colocada a 10,76 mm ( $\mathrm{i}=15$ ) y M16 colocada a 12,01 mm ( $i=16)$. Se utilizan diferentes conjuntos de coeficientes (aj, con j = 0-4) para el día y la noche y para 17 tipos de superficie de los mapas de clasificación del Programa Internacional de Geosfera-Biosfera (IGBP) (Guillevic et al., 2014; Liu et al., 2015).

La línea de base es un algoritmo de ventana dividida (SW) que aplica temperaturas de brillo medidas en las bandas VIIRS M15 y M16 centradas en longitudes de onda de 10,8 $\mathrm{m}$ y 12,0 $\mu \mathrm{m}$, respectivamente (Sun \& Pinker, 2003; Schroeder \& Giglio, 2017). El algoritmo opcional de ventana de doble división (DSW) aplica dos bandas infrarrojas de onda corta adicionales M12 y M13 centradas en longitudes de onda de 3,75 $\mu \mathrm{m}$ y 4,0 $\mu \mathrm{m}$, respectivamente. Desde el 11 de agosto el producto opera utilizando un algoritmo de ventana dividida única, que es insensible a la radiación solar (Yu et al., 2005. Se utiliza el algoritmo nocturno Nighttime DSW (Ecuación 1):

$$
\mathrm{LST}_{\mathrm{i}}=\mathrm{b}_{0}(\mathrm{i})+\mathrm{b}_{1}(\mathrm{i}) \mathrm{T}_{11}++\mathrm{b}_{2}\left(\mathrm{~T}_{11}-\mathrm{T}_{12}\right)+\mathrm{b}_{1 .}(\mathrm{i})(\sec \theta-1)+\mathrm{b}_{4}(\mathrm{i}) \mathrm{T}_{3.75}+\mathrm{b}_{5}(\mathrm{i}) \mathrm{T}_{4.0}+\mathrm{b}_{6}(\mathrm{i}) \mathrm{T}_{3.75}{ }^{2}+\mathrm{b}_{7}(\mathrm{i}) \mathrm{T}_{4.0}{ }^{2}+\mathrm{b}_{8}(\mathrm{i})\left(\mathrm{T}_{11}-\mathrm{T}_{12}\right)^{2}
$$

donde i es el índice de 17 tipos de superficies IGBP; aj (i), bj (i) y cj (i) son los coeficientes de regresión del algoritmo en los que j representa la posición secuencial del término en la ecuación; T3.75, T4.0, T11 y T12 son las temperaturas de brillo de las bandas VIIRS M12, M13, M15 y M16, respectivamente; $\theta$ es el ángulo cenital del satélite y $\varphi$ es el ángulo cenital solar. El algoritmo DSW consta de versiones diurnas y nocturnas, y se aplicó como algoritmo de referencia antes del 10 de agosto de 2012.

La elaboración de la cartografía final (termografías) para las diferentes provincias analizadas es realizada con el promedio de 50 mapas diarios a través del software de licencia gratuita QGIS 3.10.7. Se trata de 50 madrugadas estables (con procesos de inversión térmica nocturna), sin cobertura nubosa (0\%), y complementadas con el apoyo del análisis de datos de temperaturas mínimas superficiales durante los periodos caniculares de observación (2017, 2018, 2019 y 2020).

La validación estadística de Band I5 es efectuada mediante un modelo lineal de regresión con las temperaturas registradas en las estaciones meteorológicas. Es llevada a cabo para diferentes umbrales altitudinales y térmicos mediante la función "Extract Multi Values to point" del software ArcGis 10.3. A través de la exportación de ficheros de texto plano (txt) se obtiene información de la altitud y la temperatura de la termografía promedio generada para el pixel de cada una de las 147 estaciones meteorológicas utilizadas

Se utiliza el test estadístico de Shapiro Wilk para contrastar la normalidad (distribución normal o no) de un conjunto de datos (Shapiro, 1998). Si la muestra es normal se aplica el test de correlación lineal de Pearson (Eq. 2):

$$
W=1 / n 2\left(\sum^{h}{ }_{j=1} a_{i n}\left(x_{n}-i+1-x_{i}\right)^{2}\right.
$$

donde: $\mathrm{s}^{2}$ es la varianza muestral, y los coeficientes $\mathrm{a}_{\text {in }}$ suelen aparecer tabulados en los manuales.

Por el contrario, si la muestra analizada presenta asimetría y no presenta una distribución normal, se aplica el test no paramétrico del coeficiente de correlación de Spearman. Dicho test examina la fuerza y la dirección de la relación monótona entre dos variables continuas u ordinales. El valor del coeficiente de correlación puede variar de -1 a +1. Cuanto mayor sea el valor absoluto del coeficiente, más fuerte será la relación entre las variables. Para calcular $\rho$, los datos son ordenados y reemplazados por su respectivo orden (Eq. 3):

$$
\mathrm{rR}=\frac{1-\left(6 \sum \mathrm{i} \mathrm{di} 2\right)}{\mathrm{n}(\mathrm{n} 2-1)}
$$

donde $\mathrm{n}=$ número de puntos de datos de las dos variables y di= diferencia de rango del elemento " $\mathrm{n}$ "

El procedimiento aplicado minimiza simultáneamente dos funciones de costo definidas como el sesgo (BIAS) y el error cuadrático medio (RMSE) entre la LST simulada de Band I5 y la temperatura observada. El error cuadrático medio o root mean square deviation (RMSE), que mide la cantidad de error existente entre dos conjuntos de datos, es decir, un valor predicho y un valor observado o conocido (Eq. 4) (Chai \& 
Draxler, 2014). El RMSE, por tanto, es ideal para la validación de las estimaciones de temperatura del producto Band I5 (predicho), y las temperaturas registradas en estaciones meteorológicas (observado o conocido). Este estadístico ha sido utilizado en numerosas validaciones de productos como MODIS, Band I5... (Guillevic et al., 2012; Li et al., 2014; Duan et al., 2019).

$$
\operatorname{RMSE}=\frac{\sqrt{\sum_{i=1} n\left(P_{i}+O_{i}\right)}}{\mathrm{n}}
$$

donde: $\Sigma$ es el sumatorio, $\mathrm{P}_{\mathrm{i}}$ es el valor predicho para la $\mathrm{i}^{\text {th }}$ observación en el conjunto de datos, $\mathrm{O}_{\mathrm{i}}$ es el valor observado para la ith observación en el conjunto de datos, y $n$ es el tamaño de la muestra.

El Bias es una medición de la "exactitud" del sistema de medición y representa al error sistemático del sistema. Es la contribución al error total debido a los efectos combinados de todas las fuentes de variación, conocidas o desconocidas (Voinov et al., 1993). Este estudio estadístico se resuelve a través de una prueba de hipótesis t Student de una muestra y una constante, con las siguientes hipótesis. Cuando el p value es mayor a 0.05 se acepta la hipótesis nula y podemos concluir que el sistema de medición no presenta ningún sesgo.

$$
\operatorname{Bias}\left(\wedge \theta_{s^{\prime}} \theta\right)=\mathrm{E}_{s} \mathrm{D}^{m}[\wedge \theta \mathrm{s}]-\theta
$$

donde $\mathrm{E}_{\mathrm{s}}$ denota el valor esperado sobre la distribución estadística, ${ }^{\wedge} \theta$ sirve como estimador de $\theta$ basado en cualquier dato observado.

\section{RESULTADOS}

\subsection{Validación estadística del producto VIIRS LST (BandI5)}

El producto VIIRS LST (Band I5) es sometido a una verificación estadística con las temperaturas superficiales registradas en las estaciones meteorológicas de superficie. Las correlaciones estadísticas para el conjunto de la muestra son relevantes ( $R^{2}$ de Spearman de 0,87 ), con un nivel de confianza de 0,99 (gráfico 1).

El Bias, y el RMSE de las diferencias entre Band I5 y las estaciones meteorológicas es de $-0,2$ y $1,83^{\circ} \mathrm{C}$ respectivamente para el conjunto del área de estudio. RMSE es una forma útil para analizar qué tan bien un modelo de regresión (o cualquier modelo que produzca valores predichos) es capaz de "ajustarse" a un conjunto de datos. En esta ocasión los resultados arrojan una gran similitud entre ambos conjuntos (cuadro 2).

Las correlaciones estadísticas más importantes se dan en zonas de elevada altitud, aquellos ámbitos con temperaturas medias más bajas $\left(R^{2}\right.$ de 0,76$)$. Las diferencias térmicas son las más importantes de los rangos altitudinales analizados, motivados principalmente por los procesos de inversión térmica nocturna que se localizan en valles de poca extensión territorial. El análisis nocturno efectuado arroja excelentes resultados, pues existe una mejor predicción para temperaturas mínimas (Tmin) que para las temperaturas máximas (Tmax) en el análisis de productos LST (Yang et al., 2017).

En general, y a excepción de la franja altitudinal superior a 1000 m, la gran mayoría del área de estudio presenta un Bias y RMSE no superior a $-0,51$ y $1,83^{\circ} \mathrm{C}$. La franja altitudinal más elevada $(>1000 \mathrm{~m})$ posee una menor muestra estadística (102) ante el menor número de estaciones meteorológicos en ámbitos montañosos (gráfico $2 \mathrm{~d}$.). Aun así, obtiene una correlación estadística importante $\left(\mathrm{R}^{2} 0,77\right)$ y un Bias no demasiado importante $\left(-1,28^{\circ} \mathrm{C}\right)$.

La diferencia térmica entre el producto Band I5 y las temperaturas de las estaciones meteorológicas utilizadas es de $-0,2^{\circ} \mathrm{C}$. Es decir, las imágenes de satélite infraestiman muy ligeramente las temperaturas mínimas registradas en superficie, especialmente allí donde los procesos de inversión térmica nocturna son más intensos, generalmente en zonas altitudinalmente más elevadas. Se trata, por tanto, de una aproximación realmente notable, constituyendo una herramienta esencial para el estudio de la distribución de las temperaturas nocturnas. 
Las correlaciones estadísticas en el resto de intervalos altitudinales son similares, entre 0,80 y 0,75 , con diferencias térmicas poco importantes $\left(-0,5\right.$ a $\left.-0,3^{\circ} \mathrm{C}\right)$. Entre 250 y $1000 \mathrm{~m}$. de altitud, los resultados estadísticos muestran resultados muy importantes (Cuadro 2). Sin embargo, los observatorios con altitudes inferiores a $250 \mathrm{~m}$ (con los valores más templados) registran una correlación algo inferior $(0,54)$, pero corroborada estadísticamente con un Bias y RMSE de 0,41 y $1,63^{\circ} \mathrm{C}$ (gráfico $2 \mathrm{~d}$ ). En este rango altitudinal, la diferencia de temperatura es de $0,4^{\circ} \mathrm{C}$, donde los valores estimados por el producto Band I5 infraestiman la temperatura superficial registradas por los observatorios, seguramente motivado por el efecto de las islas de calor urbana (ICU), al coincidir con el área más densamente pobladas.

Cuadro 2. BIAS, RMSE, correlación estadística y diferencia media calculadas entre VIIRS LST (Band I5) y las temperaturas registradas en estaciones meteorológicas de superficie.

\begin{tabular}{|l|c|c|c|c|c|}
\cline { 2 - 6 } & Área de estudio & Altitud $>1000 \mathrm{~m}$ & Altitud $500-1000 \mathrm{~m}$ & Altitud $250-500 \mathrm{~m}$ & Altitud $<250 \mathrm{~m}$ \\
\hline $\mathrm{N}\left(\mathrm{n}^{\circ}\right.$ muestra $)$ & 799 & 102 & 265 & 150 & 282 \\
\hline $\begin{array}{l}\text { T. Shapiro-Wilk } \\
(\mathrm{p} \text { value }<0.01)\end{array}$ & $2,2 \mathrm{e}-16$ & 0.03063 & 0.0006263 & 0.02751 & 0.3362 \\
\hline Tipo de test & Spearman & Pearson & Spearman & Pearson & Pearson \\
\hline Rho / $\mathrm{R}^{2}$ & 0.872 & 0.766 & 0.749 & 0.840 & 0.563 \\
\hline Bias & -0.22 & -1.28 & -0.31 & -0.51 & 0.41 \\
\hline RMSE & 1.83 & 2.84 & 1.73 & 1.48 & 1.63 \\
\hline Diferencia media & -0.22 & -1.27 & -0.31 & -0.51 & 0.40 \\
\hline
\end{tabular}

Fuente: Elaboración propia.

Gráfico 1. BIAS, RMSE y correlación estadística calculadas entre VIIRS LST (Band I5) y las temperaturas registradas en estaciones meteorológicas de superficie (TS). Fuente: Elaboración propia.

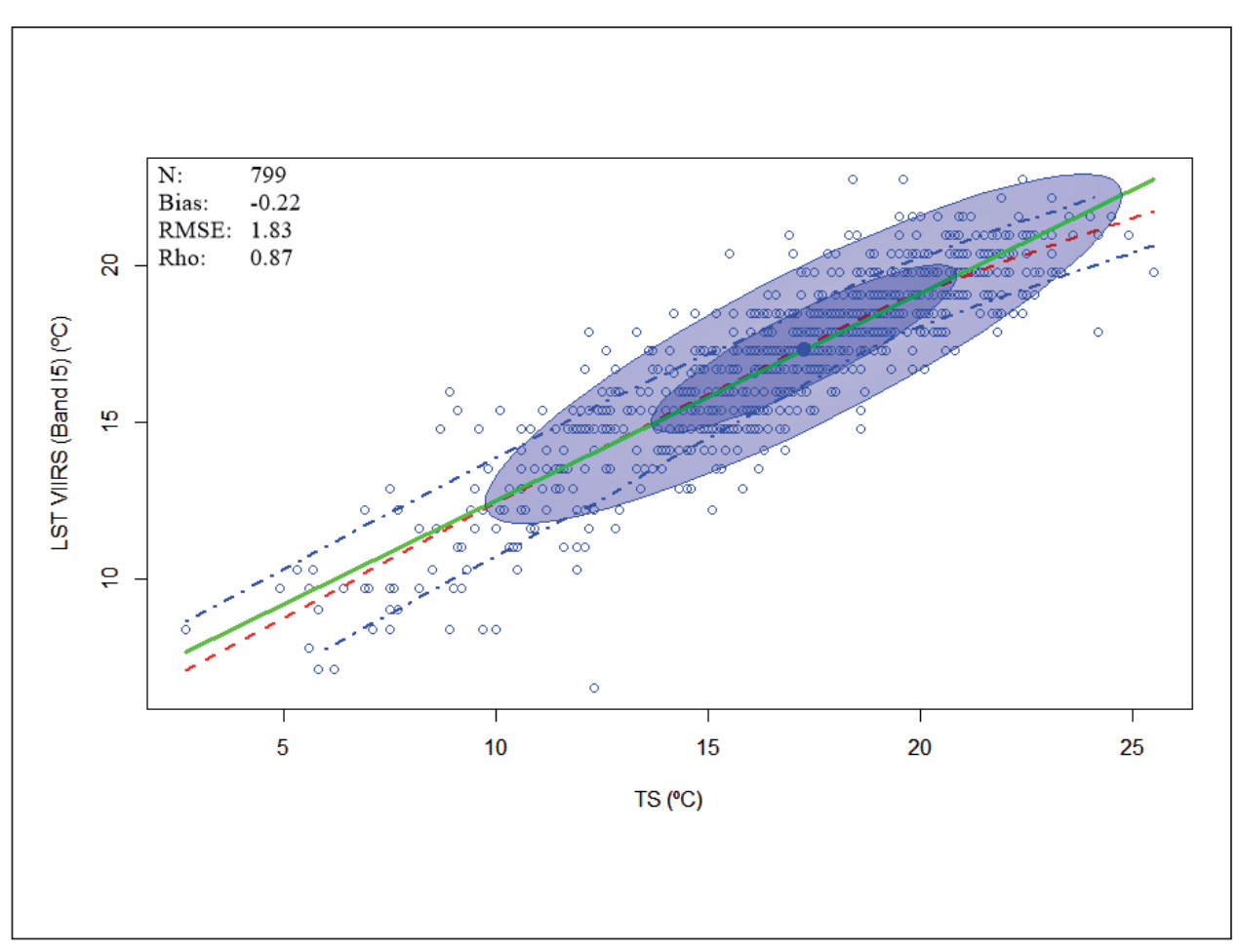




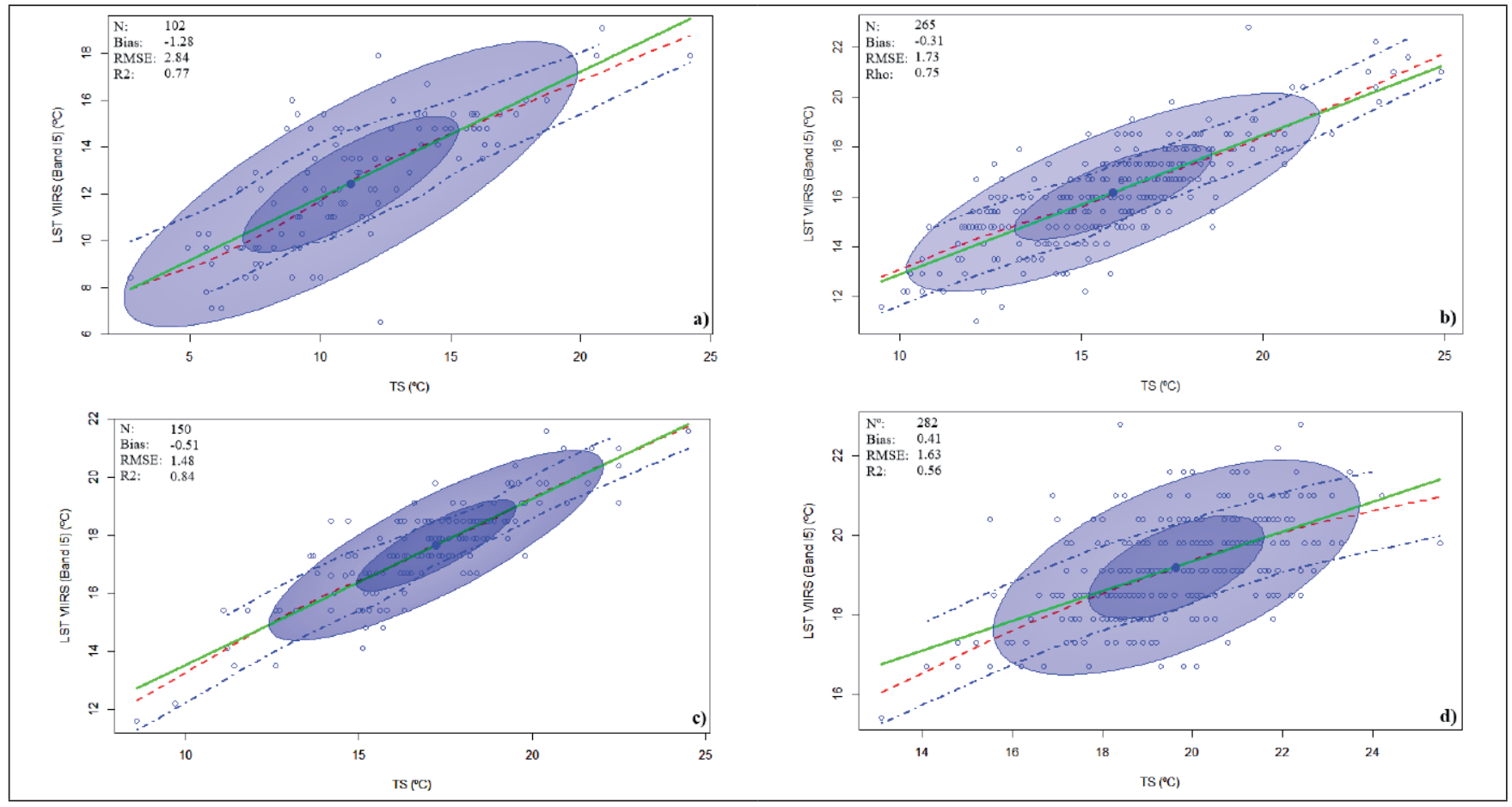

Gráfico 2. BIAS, RMSE y correlación estadística calculadas entre VIIRS LST (Band I5) y las temperaturas registradas en estaciones meteorológicas de superficie (TS) en diferentes franjas altitudinales: a) >1000 m y b) 500-1000 m, c) 250-500 m y d) <250 m. Fuente: Elaboración propia.

\subsection{Distribución espacial de las temperaturas nocturnas estivales}

\subsubsection{Provincia de Jaén}

La distribución espacial de los valores térmicos nocturnos en la provincia de Jaén viene motivada por la complejidad de la orografía. Los píxeles más elevados según el producto Band I5 aparecen en el casco urbano de Andújar $\left(21,0^{\circ} \mathrm{C}\right)$, Sierra de Andújar $\left(20,7^{\circ} \mathrm{C}\right)$, Jaén capital $\left(20,8^{\circ} \mathrm{C}\right)$ y el núcleo urbano de Linares $\left(20,8^{\circ} \mathrm{C}\right)$, donde los valores de las jornadas analizadas superan el umbral de los $20^{\circ} \mathrm{C}$ (mapa 2). La diferencia de los cascos urbanos frente a las zonas rurales limítrofes es importante, con valores que descienden a $17,0^{\circ} \mathrm{C}$ en la periferia de los cascos urbanos. Por su parte, atendiendo a los registros de las EMAs utilizadas, los valores más elevados se registran en Jaén capital $\left(21,9^{\circ} \mathrm{C}\right)$, con temperaturas de hasta $29,8^{\circ} \mathrm{C}$ (realmente notables). Otras cabeceras municipales como Bedmar o Jódar, situadas en resaltes topográficas y fueras de zonas de ITN, registran valores mínimos superiores a $28,0^{\circ} \mathrm{C}$ (cuadro 3).

Las temperaturas más bajas de la provincia (Band I5) aparecen en el Calar de Hernán Pelea (Sierra de Segura), con un valor mínimo de $8,9^{\circ} \mathrm{C}$ en el paraje del Campo del Espino, considerado en recientes publicaciones como una de las zonas más frías de la Demarcación Hidrográfica del Segura (DHS) y del sur peninsular (Espín Sánchez et al., 2018b). Las temperaturas inferiores a 10,0 ${ }^{\circ} \mathrm{C}$ aparecen en las inmediaciones de Santiago de la Espada (Vega río Zumeta), en el río Madera o en Pontones (zona occidental y de mayor altitud media de la provincia). Las temperaturas en el Calar de Magina se sitúan en $10,4^{\circ} \mathrm{C}$, o valores de $11,9^{\circ} \mathrm{C}$ al sur de la provincia, en las inmediaciones de los municipios de Alcalá la Real y Guadahortuna - Huelma.

Se trata principalmente de calares, depresiones o valles situados a altitudes superiores a $1300 \mathrm{~m}$ de altitud. Zonas de intensos procesos de ITN incluso en época estival. Las temperaturas registradas en estos sectores, a través de termómetros registradores, se sitúan en temperaturas medias de las mínimas de $8,4^{\circ} \mathrm{C}$ en la Nava del Polvo, $8,6^{\circ} \mathrm{C}$ en Monterilla, o $12,4^{\circ} \mathrm{C}$ en el núcleo poblacional de Pontones, donde los registros mínimos durante los últimos periodos caniculares son excepcionales en la mitad sur de la península ibérica, llegando a descender a $5,3^{\circ} \mathrm{C}$ en Pontones (28/07/2018). 
En sólo cuatro periodos caniculares se registra una diferencia térmica absoluta de $28,8^{\circ} \mathrm{C}$ entre Jaén capital y la Nava del Polvo, la diferencia más elevada de las provincias analizadas (gráfico 3).

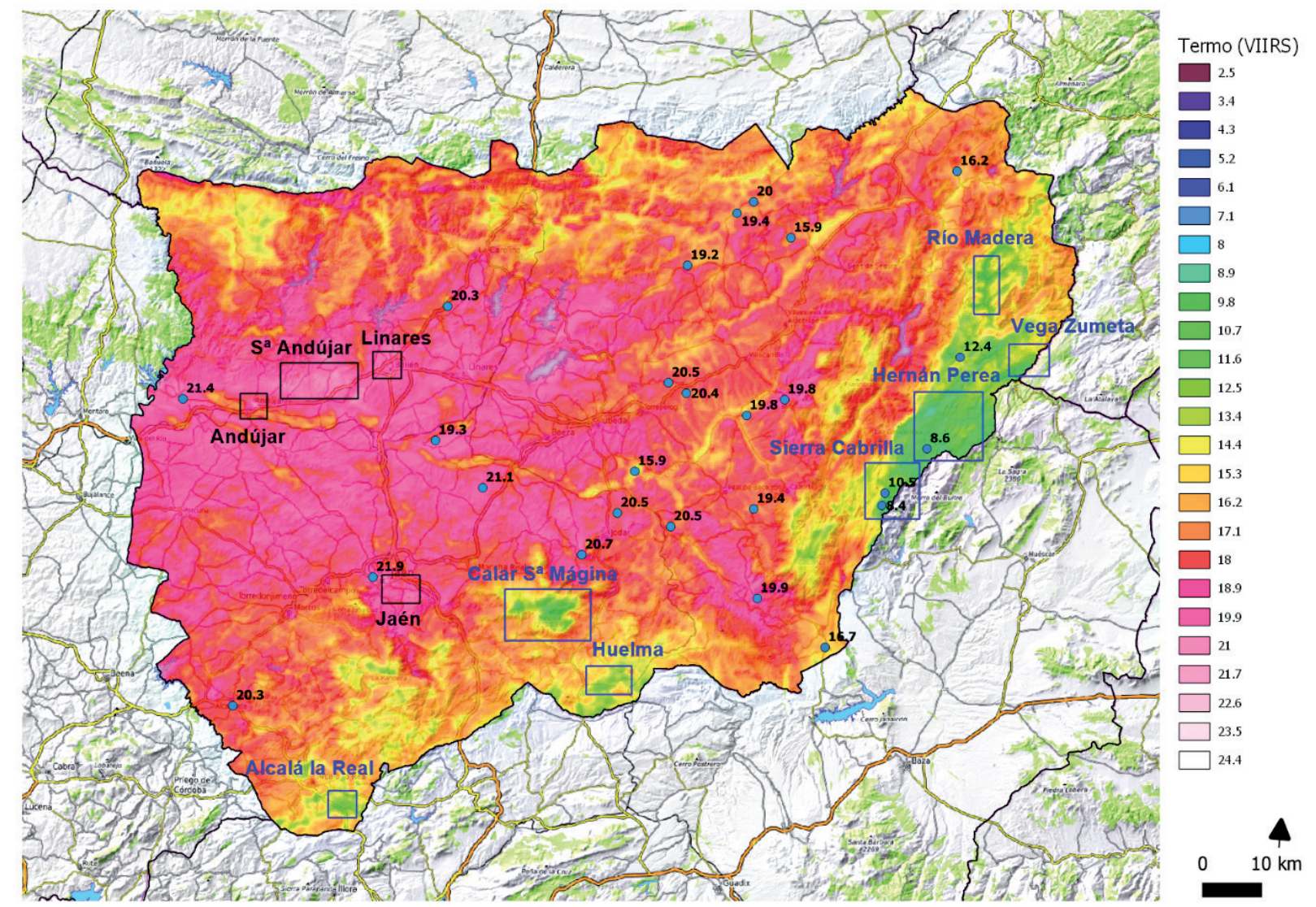

Mapa 2. Termografía elaborada a través del producto VIIRS LST (Band I5) para la provincia de Jaén. Estaciones meteorológicas utilizadas y promedio de las temperaturas mínimas durante el periodo de canícula (15 julio - 15 agosto) en el periodo temporal (2017-2020). Recuadro de color azul representa los ámbitos con los píxeles más fríos, mientras, que, los recuadros negros hacen referencia a las temperaturas más elevadas. Fuente: Elaboración propia.

Cuadro 3. Cuadro estadístico de las temperaturas mínimas diarias registradas en las estaciones meteorológicas de la provincia de Jaén durante las canículas (15 julio - 15 agosto) de 2017 a 2020.

\begin{tabular}{|l|c|c|c|}
\hline Estación meteorológica & Temperatura media de las mínimas $\left({ }^{\circ} \mathrm{C}\right)$ & Temp. mínima abs. $\left.{ }^{\circ} \mathrm{C}\right)$ & Temp. máxima de las mínimas $\left.{ }^{\circ} \mathrm{C}\right)$ \\
\hline Jaén & 21,9 & 14,6 & 29,8 \\
\hline Torreperogil & 21,5 & 15,2 & 26,0 \\
\hline Mancha Real & 21,2 & 15,4 & 25,8 \\
\hline Bedmar - Garciés & 20,8 & 13,6 & 28,3 \\
\hline Jódar & 20,7 & 14,4 & 28,3 \\
\hline Úbeda & 16,0 & 9,0 & 25,6 \\
\hline Pontones & 12,4 & 5,3 & 21,7 \\
\hline Navalasno & 10,5 & 3,3 & 19,2 \\
\hline Monterilla & 8,6 & 1,2 & 19,0 \\
\hline Nava del Polvo & 8,4 & 1,0 & 18,6 \\
\hline
\end{tabular}

Fuente: Elaboración propia. 
Gráfico 3. Diagrama de cajas o boxplot de las temperaturas mínimas registradas en cuatro estaciones meteorológicas de la provincia de Jaén durante cuatro periodos caniculares (20172020). Fuente: Elaboración propia.

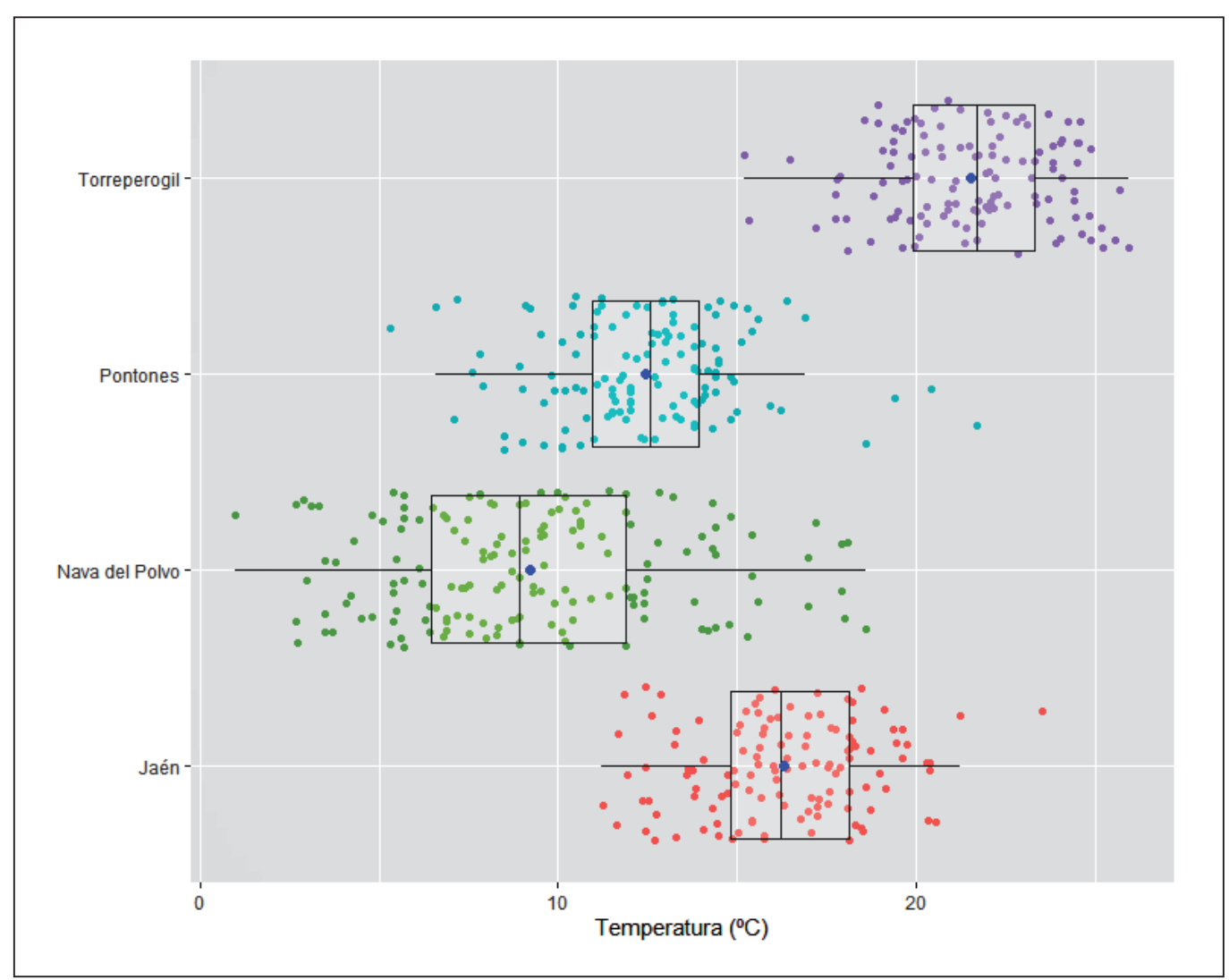

\subsubsection{Provincia de Granada}

Las temperaturas nocturnas estimadas más elevadas en la provincia de Granada (Band I5) se localizan al sur de la provincia, principalmente en el sector litoral y prelitoral. Los valores más elevados aparecen en Lobres $\left(21,9^{\circ} \mathrm{C}\right)$, Motril $\left(21,4^{\circ} \mathrm{C}\right)$, Castell de Ferro $\left(20,9^{\circ} \mathrm{C}\right)$ o Almuñecar $\left(21,0^{\circ} \mathrm{C}\right)$, o en sectores más orientales como Albuñol $\left(20,5^{\circ} \mathrm{C}\right)$ o La Rábita $\left(20,9^{\circ} \mathrm{C}\right)$. Se trata de ámbitos próximos al litoral, de escasa altitud y de orografía accidentada (Cuadro 4). Aparecen otras zonas secundarias próximas a Granada capital, por efecto de la ICU nocturna, o zonas de medianías en zonas centrales de la provincia.

Las temperaturas superficiales registradas por las EMAs muestran los valores más elevados en Motril, Ugijar o Cadiar, con temperaturas medias de las mínimas próximas a $20^{\circ} \mathrm{C}$. Se trata de ámbitos contrastados, con extremos entre 13 y $26^{\circ} \mathrm{C}$ en los periodos caniculares. Es preciso destacar que se registran valores absolutos superiores a $25^{\circ} \mathrm{C}$ en Ugijar y Cadiar.

El ámbito de alta montaña de Sierra Nevada destaca como el ámbito geográfico con las temperaturas estimadas más bajas. A pesar de su orografía accidentada, y ser poco proclive a los fenómenos de ITN, la elevada altitud (>2000 m) le otorga temperaturas mínimas inferiores a $5^{\circ} \mathrm{C}$. El pico Mulhacén obtiene el pixel más bajo de la provincia, con $2,3^{\circ} \mathrm{C}$ (mapa 3). La provincia de Granada destaca por altitudes elevadas por encima de $2000 \mathrm{~m}$ ( $\mathrm{S}^{\mathrm{a}}$ Baza, Seca, Guillimona), donde las temperaturas se sitúan entre 6,9 y $9,8^{\circ} \mathrm{C}$. Zonas habitadas como Puebla don Fadrique, en el sector septentrional de la provincia registran píxeles ligeramente superiores a $10^{\circ} \mathrm{C}$.

Los registros térmicos más bajos a través de las estaciones meteorológicas se localizan en la periferia de Guadix $\left(12,4^{\circ} \mathrm{C}\right)$, o en las inmediaciones del pojé de Zafarraya, con temperaturas mínimas absolutas inferiores a $10^{\circ} \mathrm{C}$. La temperatura mínima más baja en Guadix $\left(7.1^{\circ} \mathrm{C}\right)$ aparece el 09/08/2017.

La diferencia térmica absoluta en la provincia llega a $18,7^{\circ} \mathrm{C}$. Las distribuciones estadísticas entre los cuatro puntos analizados arrojan diferencias sustanciales, con valores del P90 en Guadix y Ugijar solapándose con el P5 de Ugijar y Motril (gráfico 4). 


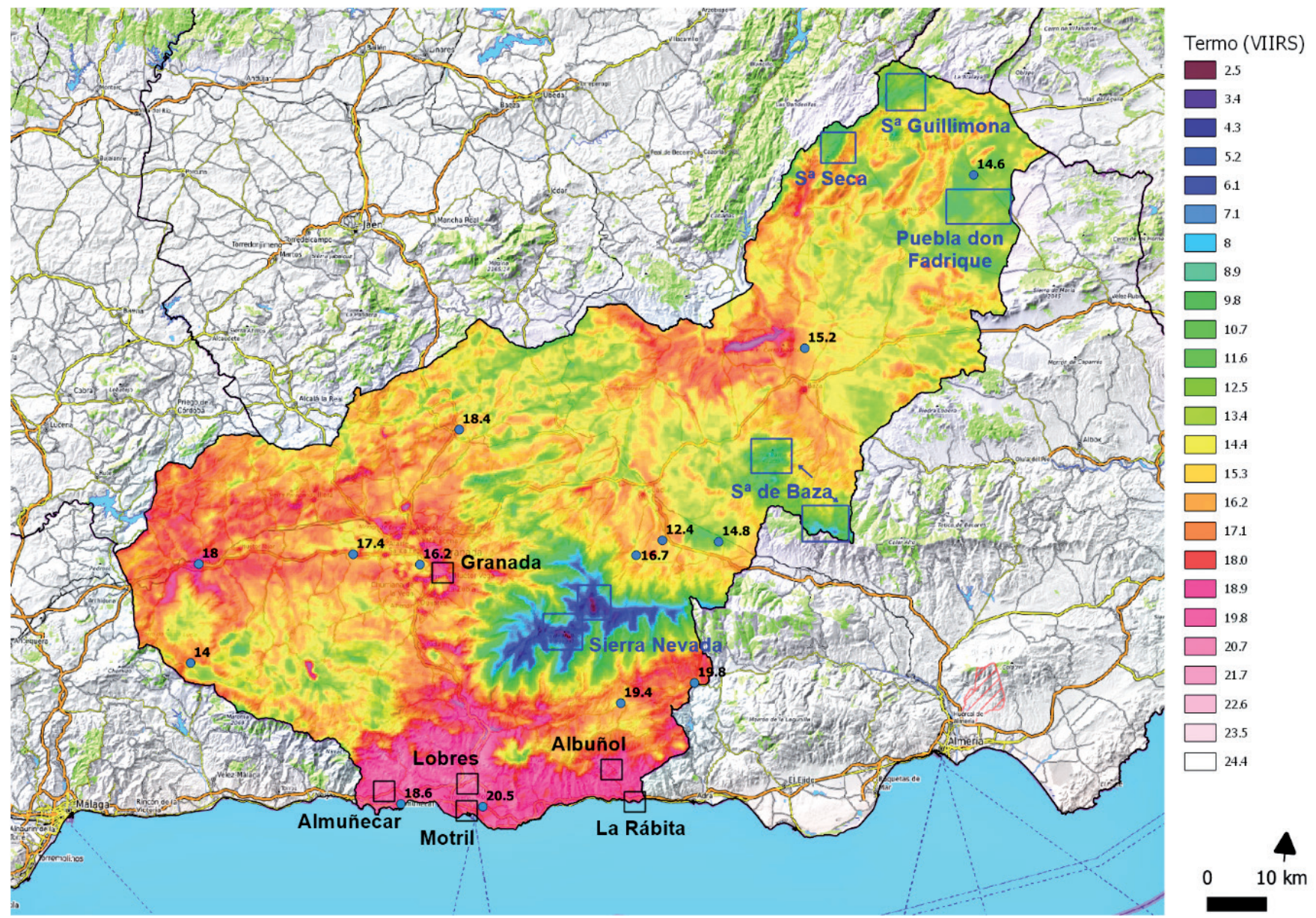

Mapa 3. Termografía elaborada a través del producto VIIRS LST (Band I5) para la provincia de Granada. Estaciones meteorológicas utilizadas y promedio de las temperaturas mínimas durante el periodo de canícula (15 julio - 15 agosto) en el periodo temporal (2017-2020). Recuadro de color azul representa los ámbitos con los píxeles más fríos, mientras, que, los recuadros negros hacen referencia a las temperaturas más elevadas. Fuente: Elaboración propia.

Cuadro 4. Cuadro estadístico de las temperaturas mínimas diarias registradas en las estaciones meteorológicas de la provincia de Granada durante las canículas (15 julio - 15 agosto) de 2017 a 2020.

\begin{tabular}{|l|c|c|c|}
\hline \multicolumn{1}{|c|}{ Estación meteorológica } & Temperatura media de las mínimas $\left(^{\circ} \mathrm{C}\right)$ & Temp. mínima abs. $\left.{ }^{\circ} \mathrm{C}\right)$ & Temp. máxima de las mínimas $\left({ }^{\circ} \mathrm{C}\right)$ \\
\hline Motril & 20,5 & 16,8 & 24,2 \\
\hline Ugijar & 19,8 & 13,8 & 25,3 \\
\hline Cadiar & 19,5 & 13,5 & 25,8 \\
\hline Almuñecar & 18,6 & 14,1 & 23,1 \\
\hline Iznalloz & 18,5 & 11,7 & 23,4 \\
\hline Baza & 15,3 & 11,0 & 20,3 \\
\hline Huéneja & 14,8 & 10,3 & 19,7 \\
\hline Puebla don Fadrique & 14,7 & 9,1 & 18,9 \\
\hline Zafarraya & 14,1 & 9,1 & 19,1 \\
\hline Guadix & 12,4 & 7,1 & 17,4 \\
\hline
\end{tabular}

Fuente: Elaboración propia. 
Gráfico 4. Diagrama de cajas o boxplot de las temperaturas mínimas registradas en cuatro estaciones meteorológicas de la provincia de Granada durante cuatro periodos caniculares (20172020). Fuente: Elaboración propia.

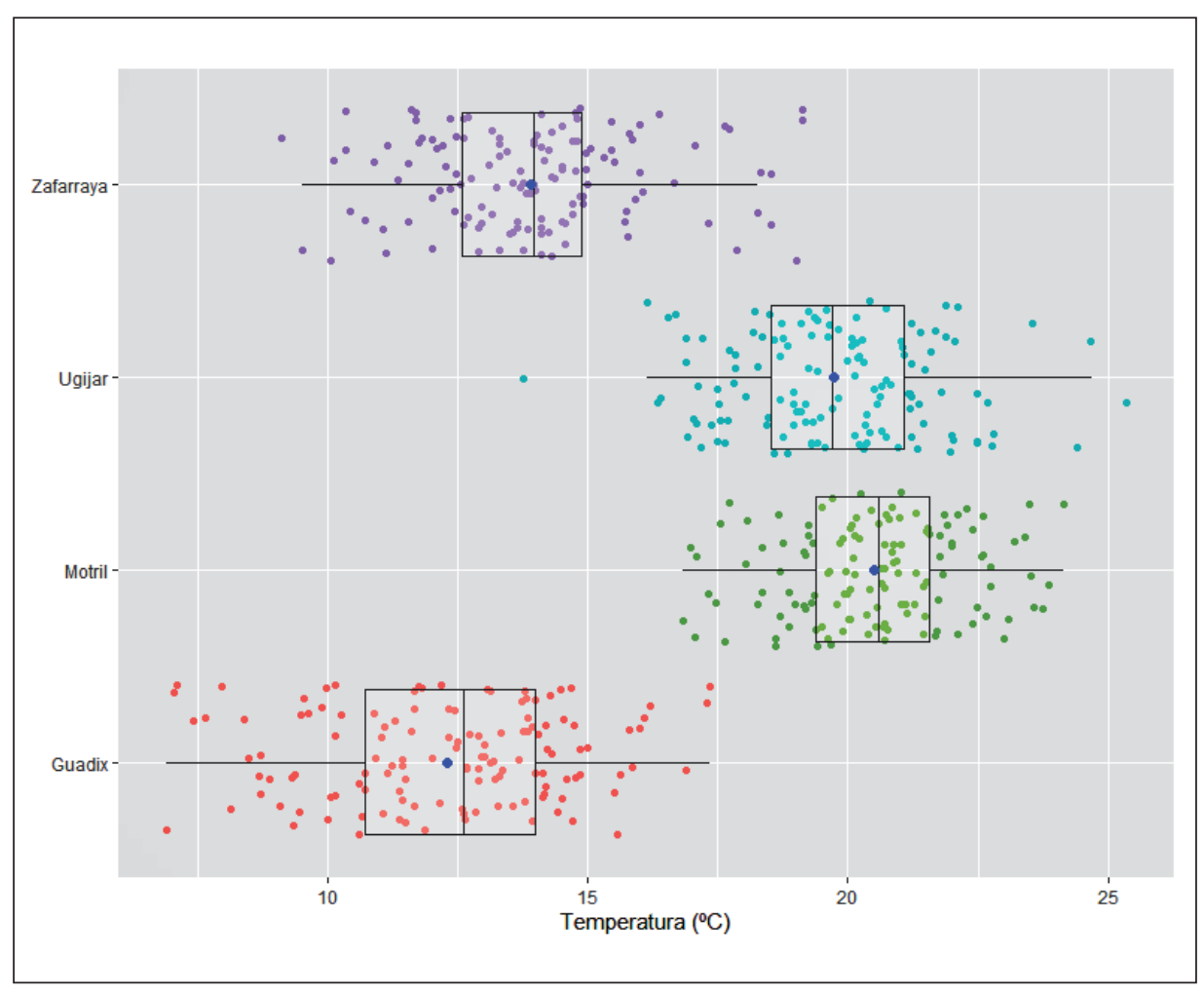

\subsubsection{Provincia de Almería}

La distribución térmica en la provincia de Almería se caracteriza por una importante dicotomía entre zonas litorales y prelitorales mediterráneas, y zonas montañosas de grandes altitudes en ámbitos de interior. Las temperaturas nocturnas más elevadas (Band I5) aparecen en la costa mediterránea, concretamente en San Juan de los Terreros $\left(22,1^{\circ} \mathrm{C}\right.$ ) y el puerto de la ciudad de Almería $\left(22,0^{\circ} \mathrm{C}\right)$, temperaturas medias más elevadas de las provincias analizadas. Otros ámbitos litorales y prelitorales (de orografía accidentada) registran temperaturas superiores a los $20^{\circ} \mathrm{C}$, como es el caso de Roquetas de $\operatorname{Mar}\left(21,2^{\circ} \mathrm{C}\right)$, el casco urbano de El Ejido $\left(20,8^{\circ} \mathrm{C}\right)$ o Huércal de Almería con $20,5^{\circ} \mathrm{C}$.

Los datos observaciones aportados por las EMAs corroboran lo cálidas de estas temperaturas nocturnas estivales, con temperaturas medias de las mínimas muy elevadas, como es el caso de Campohermoso $\left(23,5^{\circ} \mathrm{C}\right)$, o San Isidro $\left(22,2^{\circ} \mathrm{C}\right)$. Los datos aportados por el observatorio de Almería capital $\left(22,2^{\circ} \mathrm{C}\right)$ es similar al estimado por el producto Band I5 $\left(22,0^{\circ} \mathrm{C}\right)$.

En éstos ámbitos más cálidos, las temperaturas mínimas absolutas más elevadas llegan a superar 27 a $28^{\circ} \mathrm{C}$, con el valor mínimo más elevado registrado en Níjar $\left(28,8^{\circ} \mathrm{C}\right.$ ) el 23/07/2019 (Cuadro 5).

Por su parte, los valores más fríos estimados por el producto BandI5 son enormemente contrastados con los píxeles más cálidos. En ese sentido destacan los principales sistemas montañosos, tales como la Sierra de Gádor $\left(6,9^{\circ} \mathrm{C}\right)$, el ámbito almeriense de Sierra Nevada $\left(7,1^{\circ} \mathrm{C}\right)$, Sierra de los Filabres - Calar Alto $\left(8,3^{\circ} \mathrm{C}\right), 0$ los principales altiplanos a una cota altitudinal de $1000 \mathrm{~m}$. Aquí destaca el Altiplano de María $\left(10,7^{\circ} \mathrm{C}\right)$ o de Chirivel $\left(10,3^{\circ} \mathrm{C}\right)$ al norte de la provincia (Mapa 4$)$.

En éstos dos últimos ámbitos geográficos, los datos arrojados por las estaciones meteorológicas en superficie (Cañada Grande y Chirivel) registran temperaturas medias de las mínimas de 13,5 y $15,8^{\circ} \mathrm{C}$, con valores mínimos absolutos inferiores a $10^{\circ} \mathrm{C}$. Destaca la temperatura mínima de $7,4^{\circ} \mathrm{C}$ en Cañada Grande el 17/07/2018. Es preciso destacar que este paraje registra un P25 de $14^{\circ} \mathrm{C}$, mientras que, la diferencia con Campohermoso es muy significativa, con un P25 de $24^{\circ} \mathrm{C}$ (gráfica 5). 


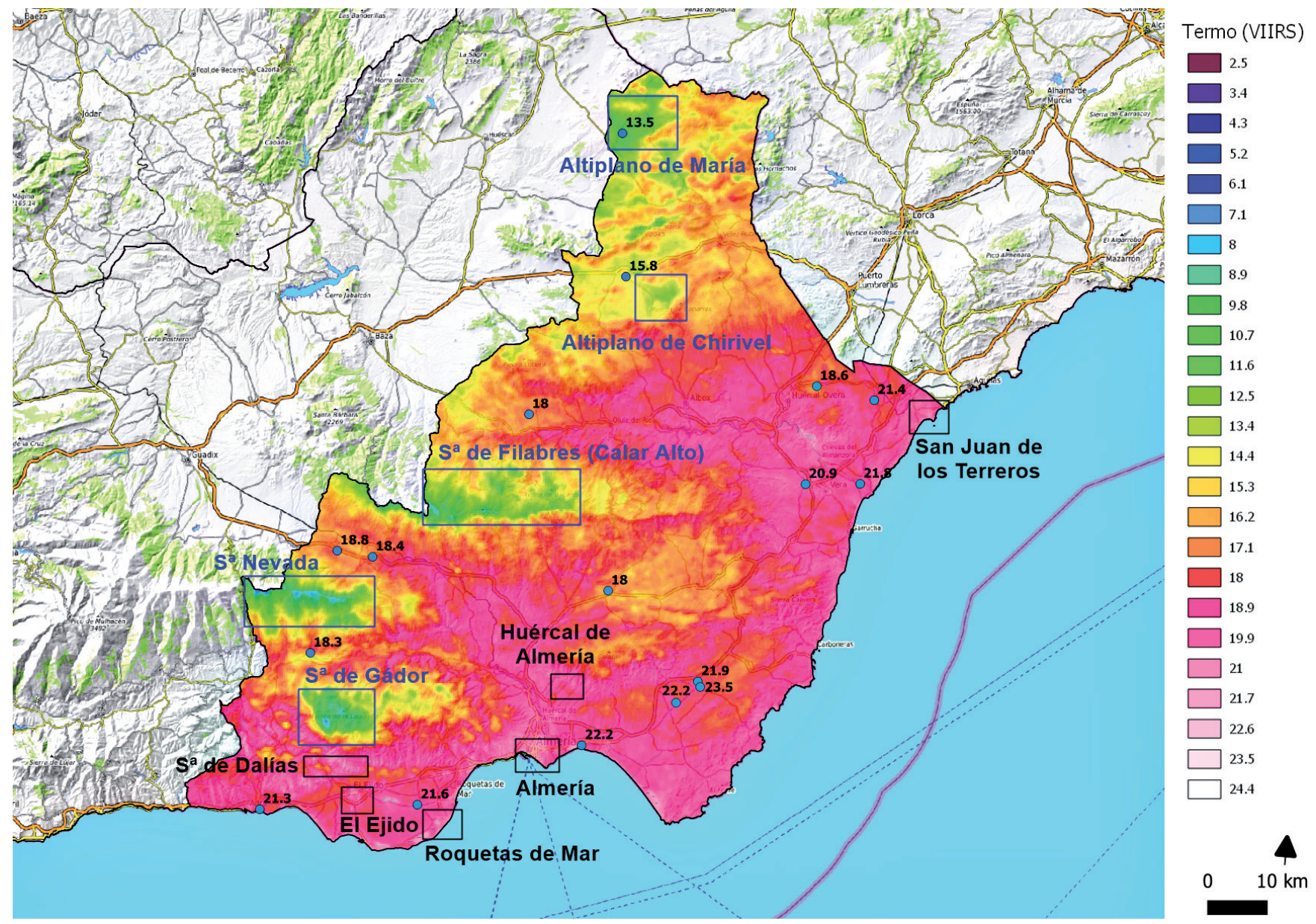

Mapa 4. Termografía elaborada a través del producto VIIRS LST (Band I5) para la provincia de Almería. Estaciones meteorológicas utilizadas y promedio de las temperaturas mínimas durante el periodo de canícula (15 julio - 15 agosto) en el periodo temporal (2017-2020). Recuadro de color azul representa los ámbitos con los píxeles más fríos, mientras, que, los recuadros negros hacen referencia a las temperaturas más elevadas. Fuente: Elaboración propia.

Cuadro 5. Cuadro estadístico de las temperaturas mínimas diarias registradas en las estaciones meteorológicas de la provincia de Almería durante las canículas (15 julio - 15 agosto) de 2017 a 2020.

\begin{tabular}{|l|c|c|c|}
\hline \multicolumn{1}{|c|}{ Estación meteorológica } & $\begin{array}{c}\text { Temperatura media de las } \\
\text { mínimas }\left({ }^{\circ} \mathrm{C}\right)\end{array}$ & $\begin{array}{c}\text { Temperatura mínima absoluta } \\
\left({ }^{\circ} \mathrm{C}\right)\end{array}$ & $\begin{array}{c}\text { Temperatura máxima de las } \\
\text { mínimas }\left({ }^{\circ} \mathrm{C}\right)\end{array}$ \\
\hline Campohermoso & 23,5 & 18,9 & 27,8 \\
\hline San Isidro & 22,2 & 15,8 & 27,4 \\
\hline Almería & 22,2 & 16,4 & 26,4 \\
\hline Níjar & 22,0 & 16,0 & 28,8 \\
\hline Cuevas de Almanzora & 21,9 & 17,6 & 25,2 \\
\hline Laujar Andarax & 18,3 & 14,0 & 23,1 \\
\hline Tabernas & 18,1 & 14,6 & 21,0 \\
\hline Tijola & 18,0 & 12,5 & 24,8 \\
\hline Chirivel & 15,8 & 9,5 & 23,7 \\
\hline Cañada Grande & 13,5 & 7,4 & 18,8 \\
\hline
\end{tabular}

Fuente: Elaboración propia. 
Gráfico 5. Diagrama de cajas o boxplot de las temperaturas mínimas registradas en cuatro estaciones meteorológicas de la provincia de Almería durante cuatro periodos caniculares (2017-2020). Fuente: Elaboración propia.

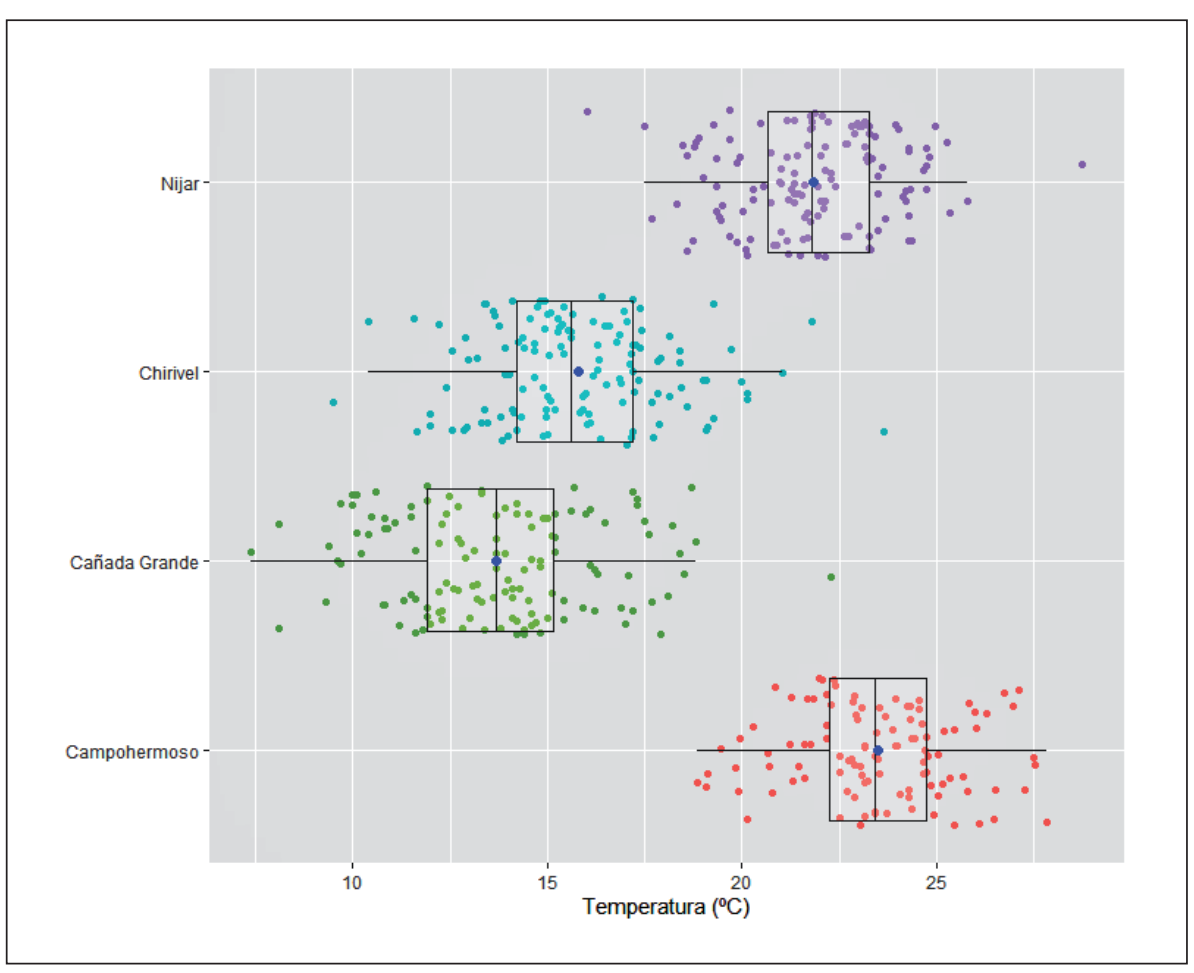

\subsubsection{Provincia de Albacete}

La provincia de Albacete se caracteriza por un menor contraste en los extremos de temperatura nocturna estival, especialmente en la amplia mayoría territorial de meseta, donde los escasos desniveles altitudinales homogeneizan las temperaturas. No es el caso del sur de la provincia, donde la orografía más accidentada genera diferencias importantes entre valles de elevada altitud y zonas de laderas.

Los píxeles más elevados según el producto Band I5 se localizan al sur de la provincia, sector geográfico accidentado de cotas altitudinalmente intermedias, donde destaca la Sierra de los Donceles $\left(19,5^{\circ} \mathrm{C}\right)$, el casco urbano de Hellín $\left(19,1^{\circ} \mathrm{C}\right)$ o el noroeste de Isso $\left(18,5^{\circ} \mathrm{C}\right)$. Aparecen también ámbitos del centro y oeste provincial, destacando la isla de calor urbana (ICU) de Albacete capital $\left(18,6^{\circ} \mathrm{C}\right)$, Caudete $\left(17,4^{\circ} \mathrm{C}\right)$ o al oeste de Villapalacios (Sierra de Relumbrar) con 18, $8^{\circ} \mathrm{C}$. Las diferencias de la ICU de Albacete capital son importantes, con píxeles entre $14-15^{\circ} \mathrm{C}$ en la periferia del casco urbano.

Las temperaturas medias de las mínimas registradas por las EMAs muestran promedios cercanos a $18^{\circ} \mathrm{C}$ en varias estaciones de la provincia. Destaca Pozo Cañada, Albacete capital o Caudete, con valores entre 17,6 y $17,8^{\circ} \mathrm{C}$. Las temperaturas mínimas absolutas descienden a $13^{\circ} \mathrm{C}$ durante las madrugadas menos cálidas del periodo de análisis, corroborando que los procesos de ITN con masas de aire frescas, generan temperaturas mínimas muy contrastadas.

Por su parte, la provincia tiene algunos sectores donde las temperaturas mínimas nocturnas son especialmente bajas. Por un lado, los valles o depresiones centrales de la provincia, donde destaca el sector Ossa de Montiel - El Bonillo $\left(11,5\right.$ a 13, $\left.0^{\circ} \mathrm{C}\right)$ o el sector norte de la Demarcación Hidrográfica del Segura (DHS) con Pétrola - Bonete $\left(12,5^{\circ} \mathrm{C}\right)$. El sector más frío aparece al sur de la provincia, en los valles y depresiones de destaca altitud de las sierras de Alcaraz, Mundo o Huebras (Mapa 5). Destaca la Vega de Riopar $\left(11,8^{\circ} \mathrm{C}\right)$, el Calar del Mundo $\left(11,4^{\circ} \mathrm{C}\right)$, Jutia $\left(11,4^{\circ} \mathrm{C}\right)$, o el pixel más frío en la depresión de Huebras $\left(10,2^{\circ} \mathrm{C}\right)$.

Respecto a las temperaturas registradas en estaciones superficiales, el valor medio de las temperaturas mínimas más bajo aparece en Viboreros, en pleno Calar del Mundo, una extensa altiplanicie ubicada a una altitud media de $1500 \mathrm{~m}$. Las temperaturas mínimas descienden a 4,9 ${ }^{\circ} \mathrm{C}(14 / 08 / 2020)$, conformando un enclave con intensos procesos de ITN, con una gran recurrencia de temperaturas mínimas inferiores a $10^{\circ} \mathrm{C}$ (Gráfica 6). Las Cañadas de Nerpio, ubicado en valle a una altitud de 1500 m, también registra un valor medio relativamente bajo $\left(13,5^{\circ} \mathrm{C}\right.$ ), con temperatura mínima absoluta de $8,7^{\circ} \mathrm{C}$ (cuadro 6 ). 


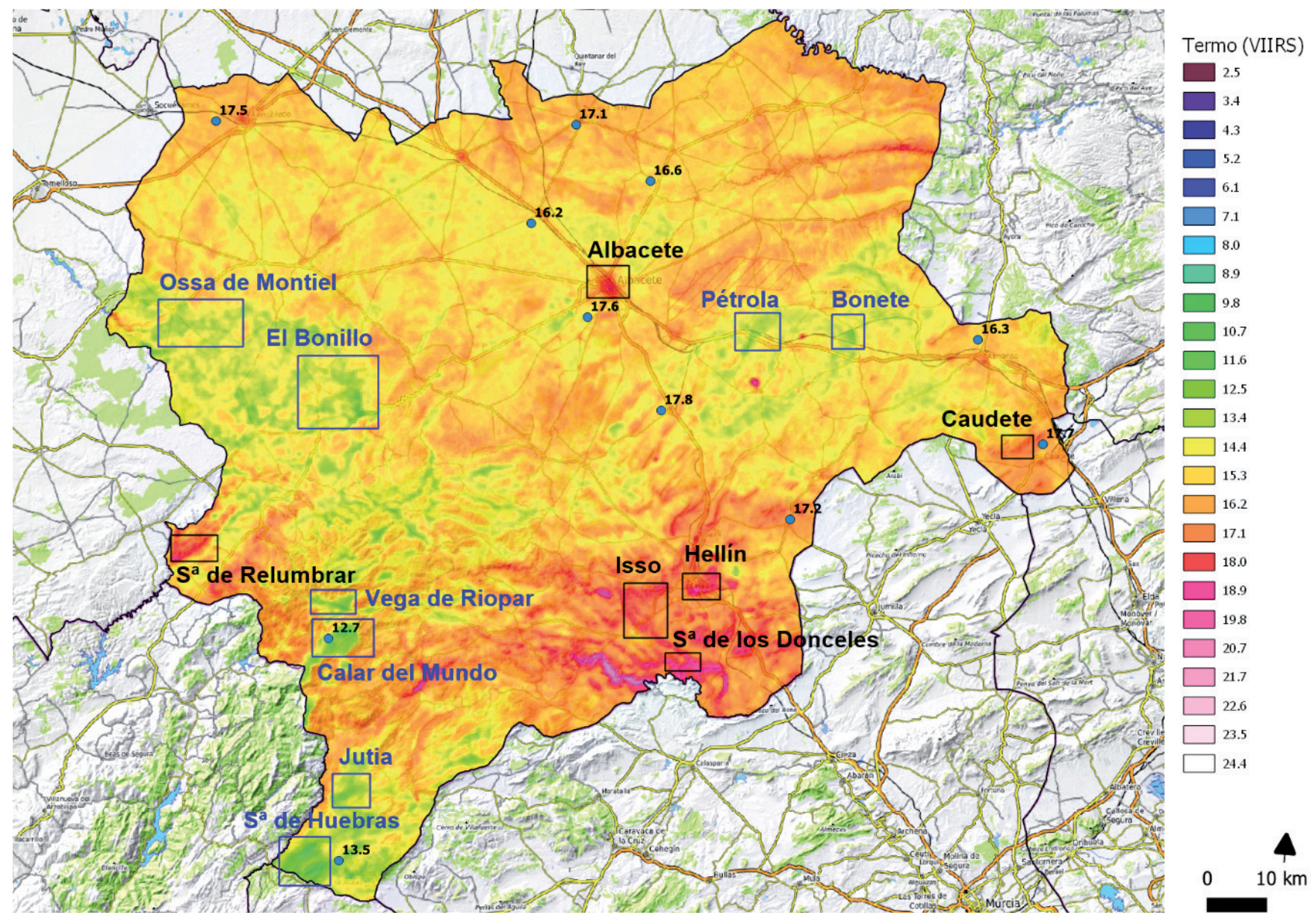

Mapa 5. Termografía elaborada a través del producto VIIRS LST (Band I5) para la provincia de Albacete. Estaciones meteorológicas utilizadas y promedio de las temperaturas mínimas durante el periodo de canícula (15 julio - 15 agosto) en el periodo temporal (2017-2020). Recuadro de color azul representa los ámbitos con los píxeles más fríos, mientras, que, los recuadros negros hacen referencia a las temperaturas más elevadas. Fuente: Elaboración propia.

Cuadro 6. Cuadro estadístico de las temperaturas mínimas diarias registradas en las estaciones meteorológicas de la provincia de Albacete durante las canículas (15 julio - 15 agosto) de 2017 a 2020.

\begin{tabular}{|l|c|c|c|}
\hline \multicolumn{1}{|c|}{ Estación meteorológica } & $\begin{array}{c}\text { Temperatura media de las } \\
\text { mínimas }(\mathrm{OC})\end{array}$ & $\begin{array}{c}\text { Temperatura mínima absoluta } \\
(\mathrm{OC})\end{array}$ & $\begin{array}{c}\text { Temperatura máxima de las } \\
\text { mínimas (oC) }\end{array}$ \\
\hline Pozo Cañada & 17,8 & 13,5 & 21,7 \\
\hline Caudete & 17,7 & 13,5 & 22,2 \\
\hline Albacete & 17,6 & 13,2 & 22,5 \\
\hline Juanaco & 17,5 & 11,8 & 22,4 \\
\hline Ontur & 17,2 & 12,4 & 21,5 \\
\hline Motilleja & 16,6 & 12,4 & 20,3 \\
\hline Almansa & 16,3 & 12,0 & 21,1 \\
\hline La Gineta & 16,2 & 12,0 & 20,0 \\
\hline Cañadas de Nerpio & 13,5 & 8,7 & 21,9 \\
\hline Viboreros & 12,7 & 4,9 & 19,9 \\
\hline
\end{tabular}

Fuente: Elaboración propia. 
Gráfico 6. Diagrama de cajas o boxplot de las temperaturas mínimas registradas en cuatro estaciones meteorológicas de la provincia de Albacete durante cuatro periodos caniculares (2017-2020). Fuente: Elaboración propia.

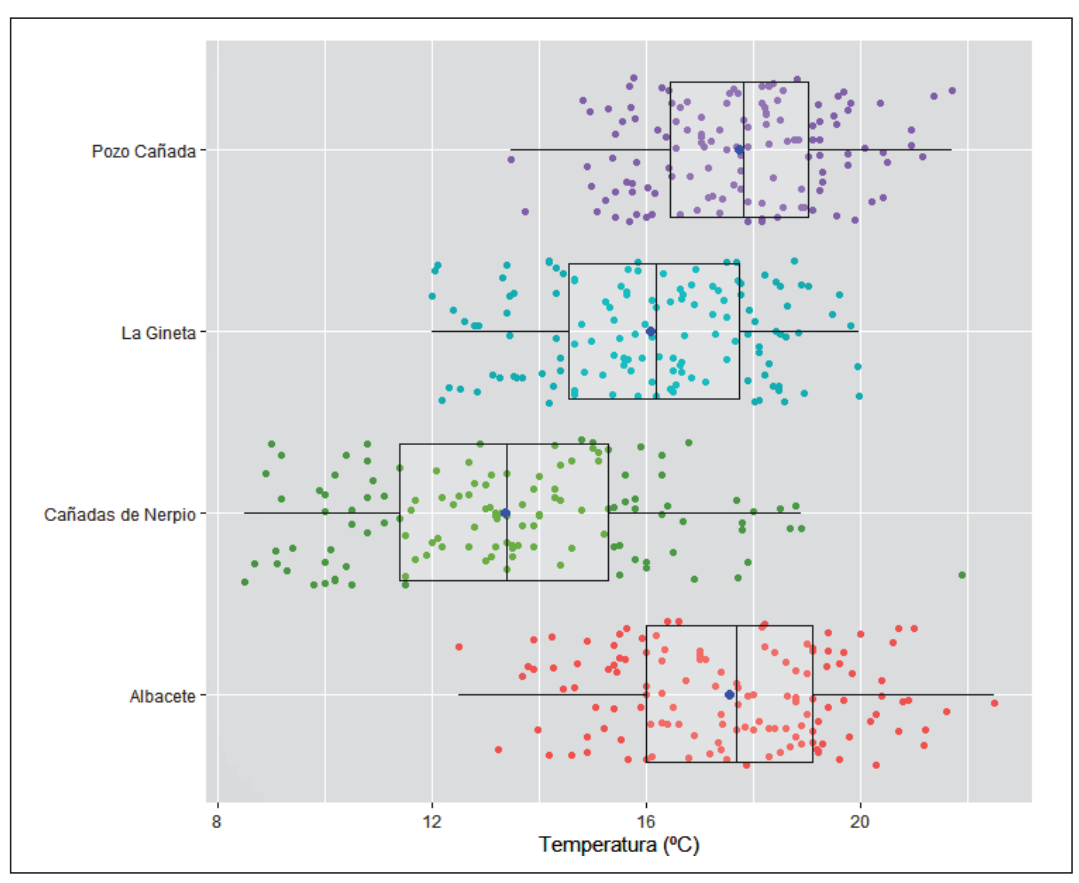

\subsubsection{Provincia de Murcia}

La provincia de Murcia se caracteriza por presentar dos ámbitos geográficos con los promedios de temperatura mínimas más bajas, al oeste de la Región (comarca del Noroeste), y la zona más septentrional (Altiplano de Murcia). El resto de la provincia registra temperaturas, por lo general, superiores a $18 / 19^{\circ} \mathrm{C}$, destacando especialmente algunos ámbitos donde las temperaturas de los píxeles (Band I5) superan los $20^{\circ} \mathrm{C}$. Destaca el área metropolitana de Murcia, donde se registran temperaturas de $21,4^{\circ} \mathrm{C}$ en el centro $021,0^{\circ} \mathrm{C}$ en el Centro Meteorológico Territorial (CMT) de AEMET. Otros municipios próximos a la capital registran valores nocturnos elevados, como Alcantarilla $\left(20,7^{\circ} \mathrm{C}\right)$, Molina de Segura $\left(20,6^{\circ} \mathrm{C}\right)$ o Archena y Ricote $\left(20,3^{\circ} \mathrm{C}\right)$. Los píxeles más elevados aparecen en el sector litoral más meridional, con valores más elevados en La Manga $\left(22,9^{\circ} \mathrm{C}\right)$, Cabo Cope $\left(22,3^{\circ} \mathrm{C}\right)$ o Cabo Tiñoso y Cartagena $\left(22,0^{\circ} \mathrm{C}\right)$ (mapa 6$)$.

Las temperaturas medias de las mínimas de las EMAs son especialmente elevadas en la ciudad de Murcia y zonas litorales. EI CMT de AEMET (Murcia) obtiene una temperatura de $23,0^{\circ} \mathrm{C}$, una de las más elevadas de las estaciones meteorológicas analizadas. En los cuatro periodos caniculares observados, la temperatura mínima absoluta es de $20,0^{\circ} \mathrm{C}$, registrando 127 madrugadas tropicales sobre 128 jornadas posibles (cuadro 7). La Pilica $\left(22,6^{\circ} \mathrm{C}\right)$ o La Alberca $\left(22,4^{\circ} \mathrm{C}\right)$, ubicados en zonas próximas a la capital de Murcia, en terrenos orográficamente accidentados, registran temperaturas medias de las mínimas superiores a $22,0^{\circ} \mathrm{C}$, al igual que ocurre en Los Belones $\left(22,6^{\circ} \mathrm{C}\right)$ o en Tébar $\left(22,1^{\circ} \mathrm{C}\right)$.

Las temperaturas mínimas son realmente contrastadas con el sector más occidental de la provincia, donde destacan valles relativamente elevados (>1000 m) muy propenso a los procesos de ITN (Mapa 6). El píxel estimado (Band I5) más bajo durante el periodo canicular aparece al sur de El Entredicho $\left(11,5^{\circ} \mathrm{C}\right)$, en el macizo de Revolcadores $\left(11,7^{\circ} \mathrm{C}\right)$, o en el altiplano de Caravaca de la Cruz, en las pedanías de El Moralejo y La Junquera $\left(11,8\right.$ a $\left.12,0^{\circ} \mathrm{C}\right)$. Se trata en general, del área con las temperaturas más bajas de la provincia, donde destacan también algunos ámbitos habitados como el Campo de San Juan $\left(12,0^{\circ} \mathrm{C}\right)$, Valle de la Rogativa $\left(12,1^{\circ} \mathrm{C}\right)$ o Calar de la Santa $\left(12,5^{\circ} \mathrm{C}\right)$.

Las temperaturas arrojadas por los termómetros registradores en el sector más occidental de la provincia son similares, destacando los valles de Gollaín, La Rogativa, Nevazo o La Risca, con promedios entre 13,5 y $13,7^{\circ} \mathrm{C}$. En estos ámbitos se registran temperaturas mínimas absolutas ligeramente inferiores a $7^{\circ} \mathrm{C}$ en pleno periodo canicular $\left(6,9^{\circ} \mathrm{C}\right.$ en La Risca el 17/07/2018), y ausencia de noches tropicales en Benablón o Gollaín, donde las temperaturas mínimas más elevadas no superan 19,3 y $18,2^{\circ} \mathrm{C}$ respectivamente. 
Las diferencias entre los extremos térmicos son notables, con temperaturas mínimas absolutas promedios que rozan $10^{\circ} \mathrm{C}$ entre el CMT de Murcia y La Risca. El gráfico 7 muestra la importante diferencia entre el P25 de las temperaturas mínimas del CMT de Murcia $\left(21.7^{\circ} \mathrm{C}\right)$ y el P95 de La Risca $\left(14.8^{\circ} \mathrm{C}\right)$.

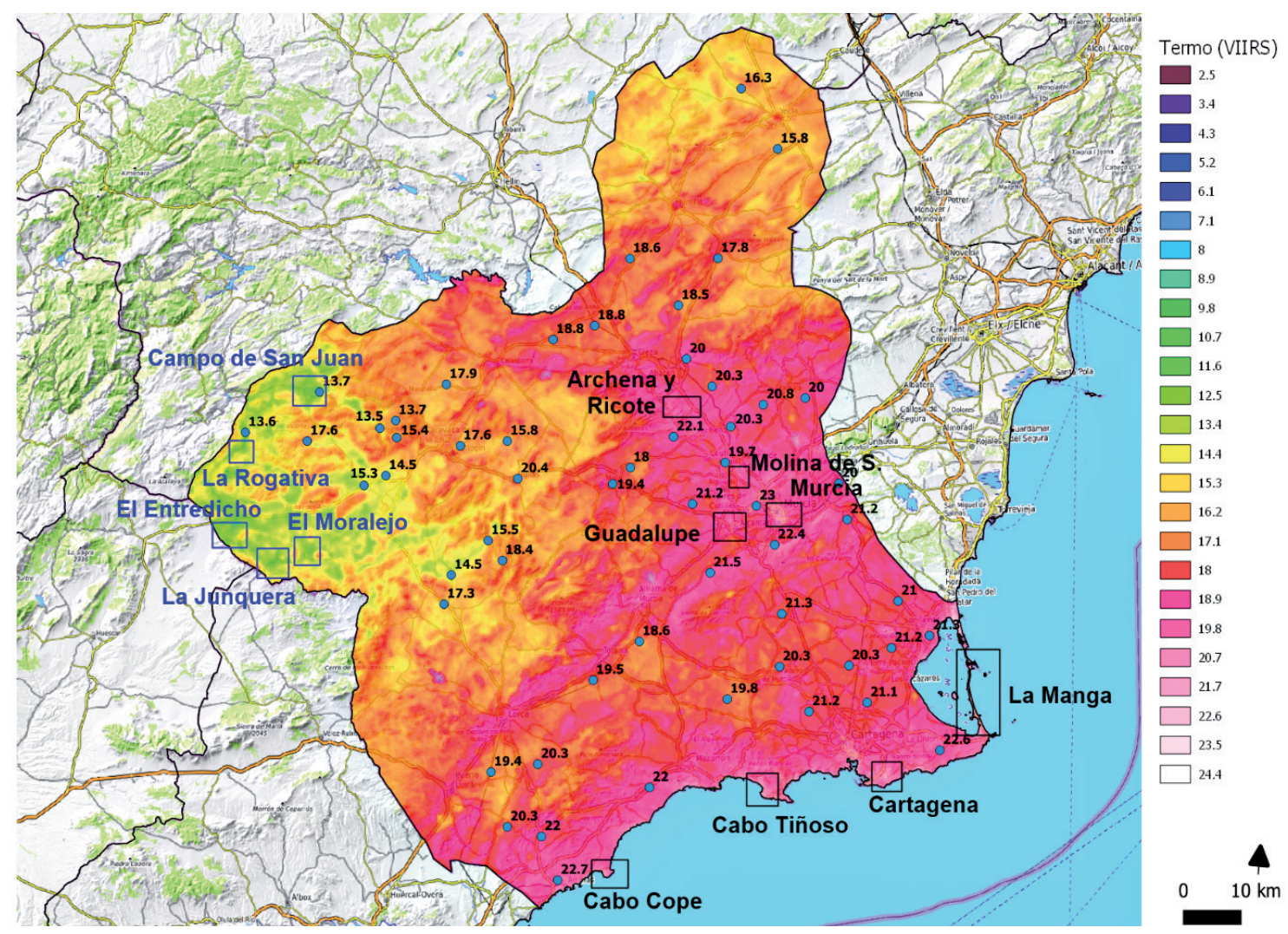

Mapa 6. Termografía elaborada a través del producto VIIRS LST (Band I5) para la provincia de Murcia. Estaciones meteorológicas utilizadas y promedio de las temperaturas mínimas durante el periodo de canícula (15 julio - 15 agosto) en el periodo temporal (2017-2020). Recuadro de color azul representa los ámbitos con los píxeles más fríos, mientras, que, los recuadros negros hacen referencia a las temperaturas más elevadas. Fuente: Elaboración propia.

Cuadro 7. Cuadro estadístico de las temperaturas mínimas diarias registradas en las estaciones meteorológicas de la provincia de Murcia durante las canículas (15 julio - 15 agosto) de 2017 a 2020.

\begin{tabular}{|l|c|c|c|}
\hline \multicolumn{1}{|c|}{ Estación meteorológica } & $\begin{array}{c}\text { Temperatura media de las } \\
\text { mínimas }\left({ }^{\circ} \mathrm{C}\right)\end{array}$ & $\begin{array}{c}\text { Temperatura mínima absoluta } \\
\left({ }^{\circ} \mathrm{C}\right)\end{array}$ & $\begin{array}{c}\text { Temperatura máxima de las } \\
\text { mínimas }\left({ }^{\circ} \mathrm{C}\right)\end{array}$ \\
\hline CMT Murcia & 23,0 & 20,0 & 26,5 \\
\hline Los Belones & 22,6 & 18,1 & 26,2 \\
\hline La Pilica & 22,6 & 18,6 & 27,1 \\
\hline La Alberca & 22,4 & 19,8 & 26,2 \\
\hline Tébar & 22,1 & 19,0 & 25,5 \\
\hline Benablón & 14,5 & 10,0 & 19,3 \\
\hline La Risca & 13,7 & 6,9 & 20,5 \\
\hline El Nevazo & 13,7 & 7,2 & 22,1 \\
\hline La Rogativa & 13,6 & 9,5 & 19,1 \\
\hline Gollaín & 13,5 & 8,3 & 18,2 \\
\hline
\end{tabular}

Fuente: Elaboración propia. 
Gráfico 7. Diagrama de cajas o boxplot de las temperaturas mínimas registradas en cuatro estaciones meteorológicas de la provincia de Murcia durante cuatro periodos caniculares (2017-2020). Fuente: Elaboración propia.

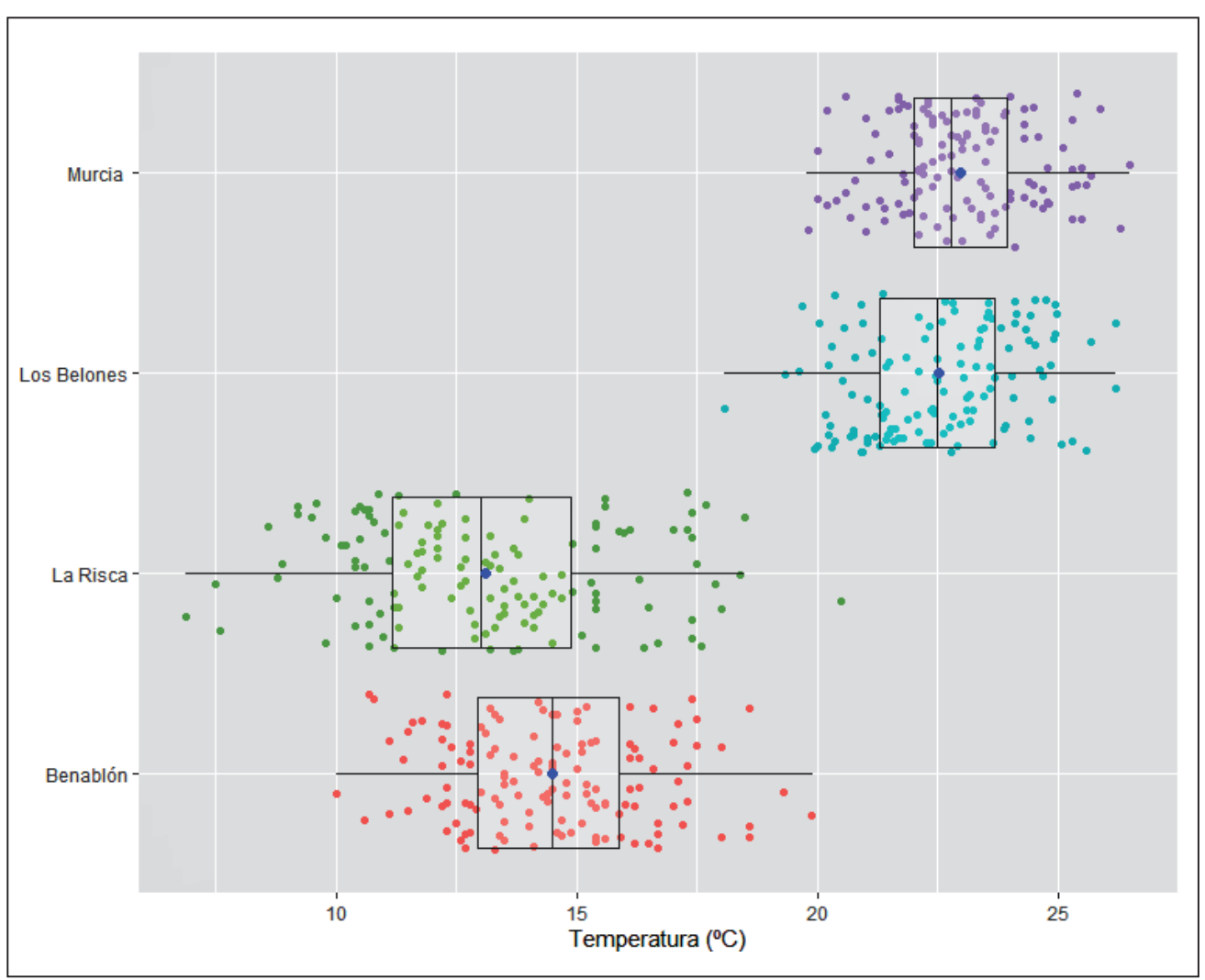

\subsubsection{Provincia de Alicante}

La provincia de Alicante posee una orografía similar a Murcia, lo que determina un litoral cálido durante las noches estivales, y un interior de mayor altitud media y multitud de valles, que le otorgan un carácter menos tórrido.

Los píxeles más elevados según el producto Band I5 se sitúan en el litoral más meridional, como Cabo Roig (Orihuela) con $22,1^{\circ} \mathrm{C}$, Torrevieja $\left(21,9^{\circ} \mathrm{C}\right)$, puerto de Alicante $\left(21,8^{\circ} \mathrm{C}\right)$ o Santa Pola $\left(21,8^{\circ} \mathrm{C}\right)$. También el litoral más septentrional como Denia registra valores medios de $21,3^{\circ} \mathrm{C}$. Finalmente, algunos ámbitos prelitorales como Crevillente y Elche registran valores medios de $21,1^{\circ} \mathrm{C}$ (mapa 7).

Las temperaturas registradas por las EMAs son similares a las estimadas por las termografías, destacando los valores medios en Crevillente $\left(22,7^{\circ} \mathrm{C}\right)$, Alicante $\left(22,6^{\circ} \mathrm{C}\right)$ o Elche $\left(22,4^{\circ} \mathrm{C}\right)$, donde las temperaturas mínimas absolutas difícilmente descienden de $20^{\circ} \mathrm{C}$ (Cuadro 8). Las temperaturas mínimas absolutas más elevadas llegan a registrar más de $27^{\circ} \mathrm{C}$ en Crevillente, Alicante o Villajoyosa.

El interior orográficamente más accidentado es el que registra las temperaturas nocturnas estivales más bajas de la provincia. Destaca, como pixel más frío (Band I5), Banyeres de Mariola $\left(13,7^{\circ} \mathrm{C}\right)$, mientras que, otros emplazamientos como el sur de Alcoy $\left(13,8^{\circ} \mathrm{C}\right)$, sur de Banyeres $\left(13,8^{\circ} \mathrm{C}\right)$ o el sur de Villena $\left(14,1^{\circ} \mathrm{C}\right)$ registran valores frescos en las noches estivales. Las estaciones meteorológicas automáticas utilizadas no están ubicadas en los sectores geográficos anteriormente descritas, por lo que las temperaturas medias de las mínimas son algo más elevadas $\left(>17^{\circ} \mathrm{C}\right)$. Destacan los municipios de Planes $\left(18,3^{\circ} \mathrm{C}\right)$, Pinoso $\left(18,2^{\circ} \mathrm{C}\right)$ o la más baja en Villena $\left(17,2^{\circ} \mathrm{C}\right)$. Las temperaturas mínimas absolutas, lejos de ser tan bajas como en valles de alta y media montaña, registran temperaturas mínimas absolutas de $11,7^{\circ} \mathrm{C}$ en Planes o $12,6^{\circ} \mathrm{C}$ en Villena (cuadro 8).

Las temperaturas nocturnas no son tan contrastadas como en las provincias de Murcia o Jaén, donde se han utilizado datalogger ubicados en polos fríos relevantes. Aun así, las diferencias medias alcanzan los $5,5^{\circ} \mathrm{C}$ de media entre Villena y Crevillente, con P25 de $16^{\circ} \mathrm{C}$ en Villena y $21.5^{\circ} \mathrm{C}$ en Alicante (gráfico 8 ). 


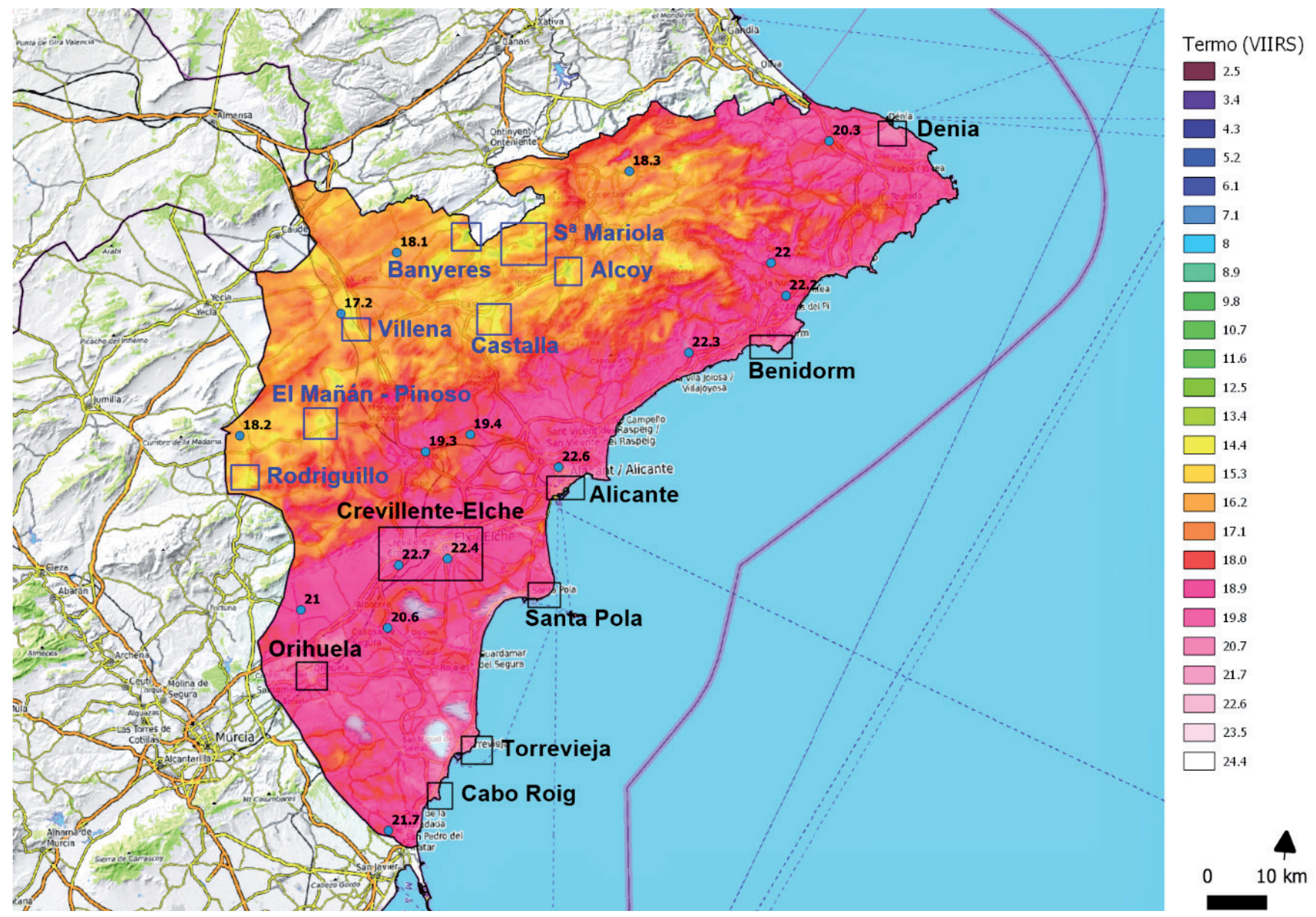

Mapa 7. Termografía elaborada a través del producto VIIRS LST (Band I5) para la provincia de Alicante. Estaciones meteorológicas utilizadas y promedio de las temperaturas mínimas durante el periodo de canícula (15 julio - 15 agosto) en el periodo temporal (2017-2020). Recuadro de color azul representa los ámbitos con los píxeles más fríos, mientras, que, los recuadros negros hacen referencia a las temperaturas más elevadas. Fuente: Elaboración propia.

Cuadro 8. Cuadro estadístico de las temperaturas mínimas diarias registradas en las estaciones meteorológicas de la provincia de Alicante durante las canículas (15 julio - 15 agosto) de 2017 a 2020.

\begin{tabular}{|l|c|c|c|}
\hline \multicolumn{1}{|c|}{ Estación meteorológica } & $\begin{array}{c}\text { Temperatura media de las } \\
\text { mínimas }\left({ }^{\circ} \mathrm{C}\right)\end{array}$ & $\begin{array}{c}\text { Temperatura mínima absoluta } \\
\left({ }^{\circ} \mathrm{C}\right)\end{array}$ & $\begin{array}{c}\text { Temperatura máxima de las } \\
\text { mínimas }\left({ }^{\circ} \mathrm{C}\right)\end{array}$ \\
\hline Crevillente & 22,7 & 19,4 & 27,3 \\
\hline Alicante & 22,6 & 17,5 & 27,2 \\
\hline Elche EFA & 22,4 & 19,6 & 25,9 \\
\hline Villajoyosa & 22,3 & 17,8 & 27,4 \\
\hline Altea & 22,2 & 17,3 & 27,1 \\
\hline Agost & 19,4 & 15,1 & 23,6 \\
\hline Planes & 18,3 & 11,7 & 22,2 \\
\hline Pinoso & 18,2 & 14,3 & 22,1 \\
\hline Camp de Mirra & 18,1 & 13,4 & 21,6 \\
\hline Villena & 17,2 & 12,6 & 20,9 \\
\hline
\end{tabular}

Fuente: Elaboración propia. 


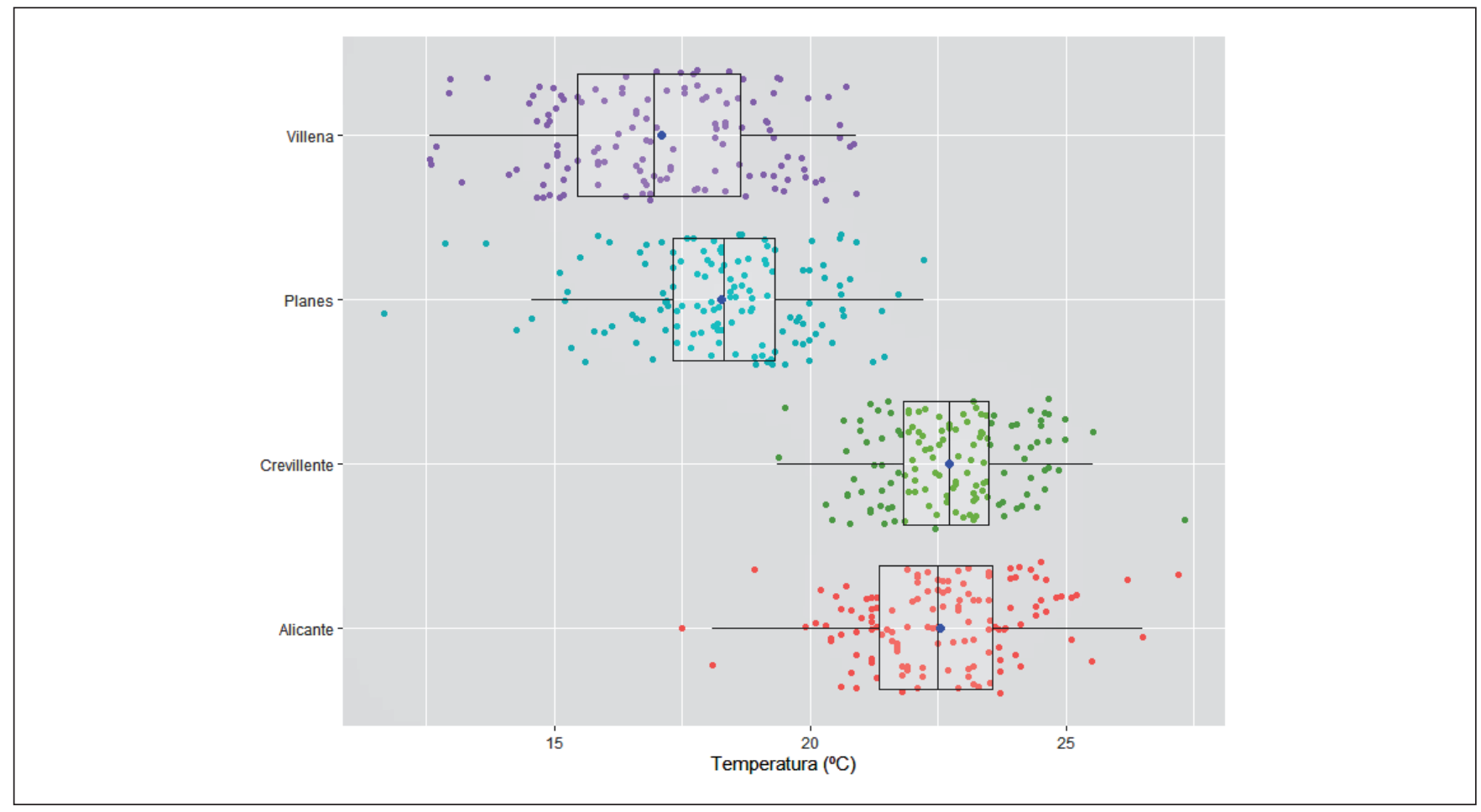

Gráfico 8. Diagrama de cajas o boxplot de las temperaturas mínimas registradas en cuatro estaciones meteorológicas de la provincia de Alicante durante cuatro periodos caniculares (2017-2020). Fuente: Elaboración propia.

\section{DISCUSIÓN Y CONCLUSIONES}

La necesidad de cartografiar los fenómenos de inversión térmica nocturna (ITN) y la distribución de las temperaturas en un territorio, ha llevado al desarrollo y proliferación de las imágenes de satélite a través de las termografías. Debido a la complejidad de la distribución espacial de temperaturas nocturnas durante madrugadas estables (conformación de CAP en fondos de valle), y a la imposibilidad de mantener una red de estaciones meteorológicas lo suficientemente densa para identificar bien el fenómeno, se utilizan las termografías nocturnas o distribución térmica a través de bandas satelitales. La validación a la que han sido sometidos diferentes productos satelitales (entre los que se encuentran Band I5) muestran que la temperatura mínima del aire puede recuperarse de forma óptima con los productos de superficie terrestre LST (Zhu et al., 2013). En ese sentido, los excelentes resultados estadísticos en la validación del producto Band I5 $(0,87)$ en los últimos años, a excepción de las ligeras discrepancias en funciones espectrales del sensor, supone una excelente relación entre las radiaciones del sensor medidas por VIIRS y la estimación de temperaturas mínimas, resultados similares a los arrojados en el oeste de EE.UU (Guillevic et al., 2014).

El producto Band I5, tras los excelentes ajustes estadísticos obtenidos en diferentes investigaciones, ha sido especialmente utilizado en los últimos años para la estimación de la temperatura superficial en diferentes regiones del planeta. Los resultados arrojados en la presente investigación, con una correlación estadística de $\mathrm{R}^{2}$ de 0,87 y diferencia térmica de $-0,22^{\circ} \mathrm{C}$ se encuentran en la horquilla de las principales investigaciones realizadas, parecidas, por ejemplo, a las de Neteler (2010), quien determina correlaciones entre 0,70 y 0,98 , y diferencias térmicas de $0,5^{\circ} \mathrm{C}$ en los Alpes centro-orientales (noreste de Italia). Los resultados también indican una precisión muy razonable de los productos actuales de VIIRS LST (Band I5), con sesgos promedio de $0,36 \mathrm{~K}$ y $-0,58 \mathrm{~K}$ y RMSE promedio de $2.7 \mathrm{~K}$ y $1.5 \mathrm{~K}$ durante el día y la noche respectivamente (Niclòs et al., 2018; Liu et al., 2015). Se trata de resultados muy ajustados a los obtenidos en el área de estudio, con un RMSE promedio de 1,83 y Bias de $-0,22^{\circ} \mathrm{C}$.

Es preciso considerar que la mayoría de la población se ubica en torno a las capitales de provincia, ejes litorales y prelitorales, donde, por lo general, las temperaturas mínimas estivales son más elevadas. Las 
capitales de provincia de Granada, Jaén o Albacete, ubicadas en zonas de interior, se ven fuertemente influenciadas por las ICUs nocturnas, con un importante incremento térmico respecto a su ámbito periurbano. Se sitúan aquí la gran mayoría de estaciones meteorológicas, por lo que los sectores con los valores promedios más elevados se encuentran bien representadas. La principal dificultad radica en la observación de áreas despobladas de interior, valles montañosos poco habitados y media/alta montaña. Aunque no ha sido posible la instalación de sensores térmicos registradores en la totalidad de la provincia, se han utilizado datalogger ubicados en los principales polos fríos de las provincias de Murcia, Jaén, Albacete y Almería (Espín Sánchez et al., 2018b). El uso de las redes propias de termómetros registradores (datalogger) ha sido ampliamente utilizada en ámbitos montañosos desprovistos de series meteorológicas. En la península, se han implementado principalmente en Picos de Europa (Cordillera Cantábrica) o Pirineos o en la plataforma kárstica del Pale di San Martino (Italia) (Renon 2011; Jiménez et al., 2015; Miró et al., 2017; Pagès et al., 2017; Iglesias González et al., 2018), válidos para multitud de investigaciones donde el análisis de temperaturas en ambientes microclimáticos es clave para el estudio de vegetación, gradientes térmicos, formación y disipación de piscinas de aire frío (CAP) y validación de imágenes de satélite (termografías).

En los últimos años, la teledetección se ha convertido en una herramienta metodológica de primer nivel para muchos campos de la climatología. La temperatura de la superficie terrestre (LST) se considera una de las formas más adecuadas para recuperar la temperatura de la superficie del aire (Ta) (Phan et al., 2019). Se ha utilizado, durante los últimos años, para evaluar el potencial TVX (Temperatura-Vegetación Index) aplicado a las imágenes térmicas MODIS con el fin de estimar las temperaturas mínimas y máximas diarias del aire en el Tibet (Pepin et al., 2019); o para el estudio de las islas de calor urbana (ICU), por ejemplo, en Madrid (Allende et al., 2018).

El análisis efectuado en el área de estudio durante la época canicular muestras notables resultados en las provincias analizadas, con una diferencia absoluta de las temperaturas medias de las mínimas de $15,1^{\circ} \mathrm{C}$ entre la Nava del Polvo $\left(8,4^{\circ} \mathrm{C}\right)$ y Los Belones $\left(23,5^{\circ} \mathrm{C}\right)$ separados por $182,2 \mathrm{~km}$ en línea recta. Y es que, en los últimos cuatro periodos caniculares (2016-2020) y un total de 128 jornadas de análisis, el Área Metropolitana de Murcia, próxima al centro de la ciudad (CMT AEMET), registra el 100\% de las madrugadas tropicales, influenciada, sin duda, por la ICU nocturna. Espín et al., (2018) determinan notables diferencias térmicas en la Vega Media del río Segura (Murcia), concretamente entre La Contraparada y el CMT de AEMET (en resalte topográfico), con un desnivel altitudinal de $4 \mathrm{~m}$ y $4,2 \mathrm{~km}$ de distancia. La tasa de cambio (lapse rate o LR) media es de $80,2^{\circ} \mathrm{C} / 100 \mathrm{~m}$, con valores absolutos de $192,9^{\circ} \mathrm{C} / 100 \mathrm{~m}$ el 06/11/2013 (temperatura mínima absoluta de $16,0^{\circ} \mathrm{C}$ en el CMT AEMET y $8,3^{\circ} \mathrm{C}$ en la Contraparada).

En el mismo ámbito temporal, las temperaturas se mantienen próximas a la helada, con valores mínimos absolutos de $1,0^{\circ} \mathrm{C}$ en la Sierra de la Cabrilla (Nava del Polvo, Jaén). La diferencia diaria más importante en temperaturas mínimas absolutas se registra el $29 / 07 / 2018$, con $23,4^{\circ} \mathrm{C}$, sin duda un valor realmente destacado. Durante la citada jornada se registra una temperatura mínima de $1,2^{\circ} \mathrm{C}$ en el paraje de Monterilla (Jaén), en el Calar de Hernán Perea, bajo un intenso proceso de ITN. En el mismo ámbito anteriormente descrito (Los Belones) la temperatura mínima no desciende de $24,6^{\circ} \mathrm{C}$. En el sondeo atmosférico de Murcia (00 h UTC) se registra una temperatura de $21,7^{\circ} \mathrm{C}$ en el geopotencial de $850 \mathrm{hPa}$, por lo que la capacidad de inversión $\left(C_{\text {INV }}\right)$ es realmente importante en Hernán Perea (Espín Sánchez et al., 2018). En general, el aire seco y templado en el nivel gepotencial de $850 \mathrm{hPa}$ favorece las intensas inversiones nocturnas. (Vihma et al., 2011), e incrementa los contrastes térmicos entre zonas propensas a ITN y resaltes orográficamente accidentados.

En definitiva, los intensos procesos de ITN en valles de elevada altitud de zonas del interior sureste peninsular, generan importantes diferencias térmicas con las zonas litorales, prelitorales, y medianías orográficamente accidentadas, donde las temperaturas mínimas estivales no suelen descender en la mayoría de ocasiones de $20^{\circ} \mathrm{C}$. La utilización de termómetros registradores en éstas zonas montañosas ha sido capaz de descubrir temperaturas mínimas inusualmente bajas en pleno periodo canicular en calares, ámbitos remotos no habitados, o valles de montaña, con temperaturas mínimas próximas a $0^{\circ} \mathrm{C}$. Incluso en zonas habitadas como La Risca o Benablón (Murcia), Cañadas de Nerpio (Albacete), Cañada Grande (Almería), Guadix (Granada) o Pontones (Jaén), las temperaturas mínimas descienden de $10^{\circ} \mathrm{C}$, por lo que se trata de una recurrencia importante en sólo 4 periodos caniculares de observación. 


\section{Agradecimientos}

Especial agradecimiento a la Agencia Estatal de Meteorología (AEMET) en su Delegación Territorial de Murcia, por la cesión de dos garitas oficiales de tipo pagoda, y por la calibración de dos termómetros registradores.

\section{Declaración responsable y conflicto de intereses}

El autor declara que no existe ningún conflicto de interés con relación a la publicación de este artículo.

\section{REFERENCIAS}

Allende, F. A., Fernández, F. G., Rasilla, D.A. \& Alcaide, J.M. (2018). Isla de calor nocturna estival y confort térmico en Madrid: avance para un planeamiento térmico en áreas urbanas. Ciudad y Territorio Estudios Territoriales (CyTET), 50(195), 101-120. https://doi.org/10.37230/CyTET.2021.M21

Anderson, M.C., Kustas, W.P., Norman, J.M., Hain, C.R., Mecikalski, J.R., Schultz, L. \& Gao, F. (2011). Mapping daily evapotranspiration at field to continental scales using geostationary and polar orbiting satellite imagery. Hydrology and Earth System Sciences, 15(1), 223-239. https://doi.org/10.5194/hess-15-223-2011

Arduini, G., Chemel, C. \& Staquet, C. (2020). Local and non-local controls on a persistent cold-air pool in the Arve River Valley. Quarterly Journal of the Royal Meteorological Society. https://doi.org/10.1002/qj.3776

Bolstad, P.V., Swift, L., Collins, F. \& Régnière, J. (1998). Measured and predicted air temperatures at basin to regional scales in the southern Appalachian Mountains. Agricultural and Forest Meteorology, 91(3-4), 161-176. https://doi. org/10.1016/S0168-1923(98)00076-8

Cao, C., Xiong, X., Wolfe, R., De Luccia, F., Liu, Q., Blonski, S.,... \& Hillger, D. (2013). Visible infrared imaging radiometer suite (VIIRS) sensor data record (SDR) user's guide. NOAA Technical Report NESDIS: College Park, MD, USA.

Chai, T. \& Draxler, R.R. (2014). Root mean square error (RMSE) or mean absolute error (MAE). Geoscientific Model Development Discussions, 7(1), 1525-1534. https://doi.org/10.5194/gmd-7-1247-2014

Clements, C.B., Whiteman, C.D. \& Horel J.D. (2003). Cold-air-pool structure and evolution in a mountain basin: Peter Sinks, Utah. Journal of Applied Meteorology, 42, 752-768. https://doi.org/10.1175/1520-0450(2003)042<0752:CSAEIA>2.0.CO;2

Daly, CH. Conklin, D.R. \& Unsworth, M.H. (2009). Local atmospheric decoupling in complex topography alters climate change impacts. International Journal of Climatology, 30(12), pp. 1857-1864. https://doi.org/10.1002/joc.2007

Dodson, R. \& Marks, D. (1997). Daily air temperature interpolated at high spatial resolution over a large mountainous region. Climate Research, 8(1), 1-20. https://doi.org/10.3354/cr008001

Duan, S.B., Li, Z.L., Li, H., Göttsche, F.M., Wu, H., Zhao, W. \& Coll, C. (2019). Validation of Collection 6 MODIS land surface temperature product using in situ measurements. Remote sensing of environment, 225, 16-29. https://doi.org/10.1016/j.rse.2019.02.020

Eisenbach, S., Pospichal, B., Whiteman, C.D., Steinacker, R. \& Dorninger, M. (2003). Classification of cold air pool events in the Gstettneralm, a sinkhole in the Eastern Alps. [Comunicación en congreso]. Extended Abstracts, Int. Conf. on Alpine Meteorology and MAP-Meeting, Brig, Switzerland, MeteoSwiss, Publication 66, 157-160.

Espín Sánchez, D. Conesa García C. \& Castejón Porcel, G. (2018a). Temperature inversions due to warm air advections at low levels: significant thermal constrast in the Vega Media of the Segura River (Southeast Spain)". In: Advances in Environmental Research Publisher: Nova Science Publishers (New York). http://doi.org/10.3989/estgeogr.201416

Espín Sánchez, D., Ruiz Álvarez, V., Martí Talavera, J. \& García Marín, R. (2018b). Estudio preliminar de las inversiones térmicas en el sureste de la Península Ibérica: El caso de los campos de Hernán Perea. Pirineos, 173, e036. https:// doi.org/10.3989/pirineos.2018.173003

Guillevic, P.C., Privette, J.L., Coudert, B., Palecki, M.A., Demarty, J., Ottle, C. \& Augustine, J.A. (2012). Land Surface Temperature product validation using NOAA's surface climate observation networks-Scaling methodology for the Visible Infrared Imager Radiometer Suite (VIIRS). Remote Sensing of Environment, 124, 282-298. https://doi.org/10.1016/j.rse.2012.05.004 
Guillevic, P.C., Biard, J.C., Hulley, G.C., Privette, J. L., Hook, S.J., Olioso, A.,... \& Csiszar, I. (2014). Validation of Land Surface Temperature products derived from the Visible Infrared Imaging Radiometer Suite (VIIRS) using ground-based and heritage satellite measurements. Remote Sensing of Environment, 154, 19-37. https://doi.org/10.1016/j.rse.2014.08.013

Gustavsson, T.; Karlsson, M.; Bogren J. \& Lindqvist, S. (1998). Development of temperature patterns during clear nights. Journal of Applied Meteorology, 37, pp. 559-571. https://doi.org/10.1175/1520-0450(1998)037<0559:DOTPDC>2.0.CO;2

Hillger D. (2013). First-light imagery from Suomi NPP VIIRS. Bull. Amer. Meteorol. Society, 94, 1019-1029. https://doi. org/10.1175/BAMS-D-12-00097.1

Hulley, G.C., Malakar, N.K., Islam, T. \& Freepartner, R.J. (2017). NASA's MODIS and VIIRS Land Surface Temperature and Emissivity Products: A Long-Term and Consistent Earth System Data Record. IEEE Journal of Selected Topics in Applied Earth Observations and Remote Sensing, 11(2), 522-535. https://10.1109/JSTARS.2017.2779330

Iglesias González, M., Acuña, J.L, García H., Rodríguez, A., Pajares, S., Rodríguez, J., Ruiz-Verdú, A. \& Jesús Pérez H. (2018): Proyecto Jous: temperaturas mínimas absolutas en la cordillera cantábrica y su relación con las piscinas de aire frío. [Comunicación en congreso]. XXXV Jornadas científicas de la AME - 19encuentro hispano-luso de meteorología. https:// doi.org/10.30859/amejrCn35

Jarvis, C.H. \& Stuart, N. (2001). A comparison among strategies for interpolating maximum and minimum daily air temperatures. Part II: The interaction between number of guiding variables and the type of interpolation method. Journal of Applied Meteorology, 40(6), 1075-1084. https://doi.org/10.1175/1520-0450(2001)040<1075:ACASFI>2.0.CO;2

Jiménez, M.A., Ruiz, A. \& Cuxart, J. (2015). Estimation of cold pool areas and chilling hours through satellite-derived surface temperatures. Agricultural and Forest Meteorology, 207, 58-68. https://doi.org/10.1016/j.agrformet.2015.03.017

Li, H., Sun, D., Yu, Y., Wang, H., Liu, Y., Liu, Q.,... \& Cao, B. (2014). Evaluation of the VIIRS and MODIS LST products in an arid area of Northwest China. Remote Sensing of Environment, 142, 111-121. https://doi.org/10.1016/j.rse.2013.11.014

Lindkvist, L.; Gustavsson, T. \& Bogren, J. (2000). A frost assessment method for mountainous areas. Agricultural and Forest Meteorology, 102, 51-67. https://doi.org/10.1016/S0168-1923(99)00087-8

Litschauer, D., (1962). Untersuchung der Entwicklung von Kaltluftseen in Dolinen- und Beckenlagen (Investigation of the development of cool air pools in sinkholes and basins) [Tesis Doctoral]. University of Vienna.

Liu, Y., Yu, Y., Yu, P., Göttsche, F. \& Trigo, I. (2015). Quality assessment of S-NPP VIIRS land surface temperature product. Remote Sensing, 7(9), 12215-12241. https://doi.org/10.3390/rs70912215

Lundquist J.D. \& Huggett B. (2008). Evergreen trees as inexpensive radiation shields for temperature sensors. Water Resour. Res. 44: W00D04. https://doi.org/10.1029/2008WR006979

McKee, T.B. \& O'Neal, R.O. (1989). The role of valley geometry and energy budget in the formation of nocturnal valley winds. Journal of Applied Meteorology, 28, 445-456. https://doi.org/10.1175/1520-0450(1989)028<0445:TROVGA>2.0.CO;2

Minnett, P.J., Evans, R.H., Podestá, G.P. \& Kilpatrick, K.A. (2014). Sea-Surface Temperature from Suomi-NPP VIIRS: Algorithm development and uncertainty estimation. [Comunicación en congreso] Ocean Sensing and Monitoring VI. International Society for Optics and Photonics (9111, p. 91110C).

Miró, J.R., Peña, J. C., Pepin, N., Sairouni, A. \& Aran, M. (2017). Key features of cold-air pool episodes in the northeast of the Iberian Peninsula (Cerdanya, eastern Pyrenees). International Journal of Climatology, 37. https://doi.org/10.1002/joc.5236

Mosiño, A.P. \& García, E. (1966). Evaluación de la sequía intraestival en la República Mexicana. Proc. Conf. Reg. Latinoamericana Unión Geogr. Int, 3, 500-516.

Nalder, I.A. \& Wein, R.W. (1998). Spatial interpolation of climatic normals: test of a new method in the Canadian boreal forest. Agricultural and forest meteorology, 92(4), 211-225. https://doi.org/10.1016/S0168-1923(98)00102-6

Neteler, M. (2010). Estimating daily land surface temperatures in mountainous environments by reconstructed MODIS LST data. Remote sensing, 2(1), 333-351. https://doi.org/10.3390/rs1020333

Niclòs, R., Pérez-Planells, L., Coll, C., Valiente, J.A. \& Valor, E. (2018). Evaluation of the S-NPP VIIRS land surface temperature product using ground data acquired by an autonomous system at a rice paddy. ISPRS Journal of Photogrammetry and Remote Sensing, 135, 1-12. https://doi.org/10.1016/j.isprsjprs.2017.10.017

Noi, P., Kappas, M. \& Degener, J. (2016). Estimating daily maximum and minimum land air surface temperature using MODIS land surface temperature data and ground truth data in Northern Vietnam. Remote Sensing, 8(12), 1002. https://doi.org/10.3390/rs8121002

Pagès, M., Pepin, N. \& Miró, J.R. (2017). Measurement and modelling of temperature cold pools in the Cerdanya valley (Pyrenees), Spain. Meteorological Applications, 2(24), 290-302 https://doi.org/10.1002/met.1630 
Pepin, N., Deng, H., Zhang, H., Zhang, F., Kang, S. \& Yao, T. (2019). An examination of temperature trends at high elevations across the Tibetan Plateau: The use of MODIS LST to understand patterns of elevation-dependent warming. Journal of Geophysical Research: Atmospheres, 124(11), 5738-5756. https://doi.org/10.1029/2018JD029798

Phan, T.N., Kappas, M., Nguyen, K.T., Tran, T.P., Tran, Q.V. \& Emam, A.R. (2019). Evaluation of MODIS land surface temperature products for daily air surface temperature estimation in northwest Vietnam. International Journal of Remote Sensing, 40(14), 5544-5562. https://doi.org/10.1080/01431161.2019.1580789

Pospichal, B., Eisenbach, S., Whiteman, C.D., Steinacker, R. \& Dorninger, M. (2003). Observations of the cold air outflow from a basin cold pool through a low pass. [Comunicación en congreso]. In: Ext. Abstr., Vol. A, Intl Conf. Alpine Meteor. and the MAP-Meeting, 153-156.

Rajasekar, U. \& Weng, Q. (2009). Urban heat island monitoring and analysis using a non-parametric model: A case study of Indianapolis. ISPRS Journal of Photogrammetry and Remote Sensing, 64(1), 86-96. https://doi.org/10.1016/j.isprsjprs.2008.05.002

Renon, B. (2011). Le fabbriche naturali del freddo. Dipartimento Regionale per la Sicurezza del Territorio. ARPAV

Sauberer, F. \& Dirmhirn, I. (1954). Über die Entstehung der extremen Temperaturminima in der Doline Gstettner-Alm". Archiv für Meteorologie, Geophysik und Bioklimatologie, Serie B, 5(3-4), 307-326. https://doi.org/10.1007/BF02242757

Sauberer, F. \& Dirmhirn, I. (1956). Weitere Untersuchungen über die kaltluftansammungen in der Doline Gstettner-Alm bei Lunz im Niederösterreich (Further investigations of the cold air buildup in the Gstettner-Alm doline near Lunz in lower Austria). Wetter Leben, 8, 187-196.

Schroeder, W. \& Giglio, L. (2017). VIIRS/NPP Thermal Anomalies/Fire 6-Min L2 Swath 750m V001. NASA EOSDIS Land Processes DAAC. https://doi.org/10.5067/VIIRS/VNP14.001

Shapiro, S.S. (1998). Distribution assessment, Statistics (17): Applications (Eds.N. Balakrishnan and C. R. Rao), pp. $475-494$. Elsevier.

Sheridan, P.F. (2019). Synoptic-flow interaction with valley cold-air pools and effects on cold-air pool persistence: Influence of valley size and atmospheric stability. Quarterly Journal of the Royal Meteorological Society, 145(721), 1636-1659. https://doi.org/10.1002/qj.3517

Sismanidis, P., Keramitsoglou, I., Bechtel, B. \& Kiranoudis, C. (2016). Improving the downscaling of diurnal land surface temperatures using the annual cycle parameters as disaggregation kernels. Remote Sensing, 9(1), 23. https://doi. org/10.3390/rs9010023

Sun, D. \& Pinker, R.T. (2003). Estimation of land surface temperature from a Geostationary Operational Environmental Satellite (GOES-8). Journal of Geophysical Research: Atmospheres, 108(D11). https://doi.org/10.1029/2002JD002422

Thornton, P.E., Running, S.W. \& White, M.A. (1997). Generating surfaces of daily meteorological variables over large regions of complex terrain. Journal of Hydrology, 190(3-4), 214-251. https://doi.org/10.1016/S0022-1694(96)03128-9

Vancutsem, C., Ceccato, P., Dinku, T. \& Connor, S.J. (2010). Evaluation of MODIS land surface temperature data to estimate air temperature in different ecosystems over Africa. Remote Sensing of Environment, 114(2), 449-465. https://doi. org/10.1016/j.rse.2009.10.002

Vihma, T., Kilpeläinen, T., Manninen, M., Sjöblom, A., Jakobson, E., Palo, T. \& Maturilli, M. (2011). Characteristics of temperature and humidity inversions and low-level jets over Svalbard fjords in spring. Advances in Meteorology, 2011. https://doi.org/10.1155/2011/486807

Voinov, Vassily G., Nikulin \& Mikhail S. (1993). Unbiased estimators and their applications. Univariate case. Dordrect: Kluwer Academic Publishers.

Wan, Z., Hook, S. \& Hulley, G. (2015). MYD11A1 MODIS/Aqua Land Surface Temperature/Emissivity Daily L3 Global 1km SIN Grid V006. NASA EOSDIS Land Processes DAAC. https://10.5067/MODIS/MYD11A1.006

Whiteman, C.D. (2000). Mountain Meteorology: Fundamentals and Applications. Oxford University Press.

Whiteman, C.D., Pospichal, B., Eisenbach, S., Weihs, P., Clements, C.B., Steinacker, R. \& Dorninger, M., (2004). Inversion breakup in small Rocky Mountain and Alpine basins. Journal of Applied Meteorology, 43(8), 1069-1082. https://doi. org/10.1175/1520-0450(2004)043<1069:IBISRM>2.0.CO;2

Whiteman, C.D., Hoch, S.W., Hahnenberge R.M., Muschinski, A., Hohreiter, V., Behn, M. \& Clements, C.B., (2008). METCRAX 2006: Meteorological experiments in arizona's meteor crater. Bulletin of the American Meteorological Society, 89(11), 1665-1680. https://doi.org/10.1175/2008BAMS2574.1 
Yang, Y., Cai, W. \& Yang, J. (2017). Evaluation of MODIS land surface temperature data to estimate near-surface air temperature in Northeast China. Remote Sensing, 9(5), 410. https://doi.org/10.3390/rs9050410

Yu, Y., Privette, J. L. \& Pinheiro, A. C. (2005). Analysis of the NPOESS VIIRS land surface temperature algorithm using MODIS data. IEEE Transactions on Geoscience and Remote Sensing, 43(10), 2340-2350. Zhou. https://doi.org/10.1117/1. JRS.11.046016

Yu, Y., Tarpley, D., Privette, J.L., Goldberg, M.D., Raja, M. R. V., Vinnikov, K.Y. \& Xu, H. (2008). Developing algorithm for operational GOES-R land surface temperature product. IEEE. Transactions on Geoscience and Remote Sensing, 47(3), 936-951. https://doi.org10.1109/TGRS.2008.2006180

Zhang Y.H., Zhang S.D., Yi F. \& Chen Z.Y. (2011). Statistics of lower tropospheric inversions over the continental United States. Ann Geophys 29, 401-410. https://doi.org/10.5194/angeo-29

Zhou, W., Peng, B. \& Shi, J. (2017). Reconstructing spatial-temporal continuous MODIS land surface temperature using the DINEOF method. Journal of Applied Remote Sensing, 11(4), 046016. https://doi.org/10.1117/1.JRS.11.046016

Zhu, W., Lü, A. \& Jia, S. (2013). Estimation of daily maximum and minimum air temperature using MODIS land surface temperature products. Remote Sensing of Environment, 130, 62-73. https://doi.org/10.1016/j.rse.2012.10.034 